\title{
The Multi-Attributive Border Approximation Area Comparison (MABAC) method for decision-making under Interval-valued Fermatean fuzzy environment for green supplier selection
}

\author{
Manoj Mathew ${ }^{1}$, Ripon K Chakrabortty ${ }^{2 *}$, Michael J. Ryan ${ }^{3}$, Muhammad Fazal Ljaz ${ }^{4}$, and Syed Abdul \\ Rehman Khan ${ }^{5}$ \\ ${ }^{1}$ Department of Mechanical Engineering, \\ Shri Shankaracharya Institute of Professional Management and Technology, \\ Mujgahan, Raipur, Chhattisgarh 492015, India \\ ${ }^{2}$ School of Engineering and Information Technology, \\ UNSW Canberra at ADFA, Canberra, Australia \\ ${ }^{3}$ Capability Associates, Canberra, Australia
}

${ }^{4}$ Department of Intelligent Mechatronics Engineering, Sejong University, Seoul 05006, South Korea

${ }^{5}$ School of Management and Engineering, Xuzhou University of Technology, Xuzhou, China

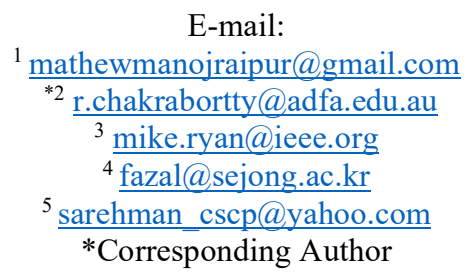

\begin{abstract}
In the era of sustainable development, green supplier selection has become a key component of supply chain management, as it considers criteria such as carbon footprint, water usage, energy usage and recycling capacity. Since the green supplier selection problem involves subjective criteria and uncertainty in preferences, it is well suited to using multi-criteria decision-making methods (MCDM). Although few researchers have investigated MCDM for green supplier selection under uncertainty using intuitionistic and Pythagorean fuzzy sets. Still, the newly developed fermatean fuzzy can handle greater data uncertainty than intuitionistic and Pythagorean fuzzy sets. Since interval extension of fuzzy theory provides more accurate modeling, in this paper, we propose an interval extension of fermatean fuzzy set and discuss its fundamental set operations, arithmetic operations and related properties. We propose the Hamming distance function and the score function of the interval-valued fermatean fuzzy numbers. The recently developed Multi-attribute Border Approximation Area Comparison (MABAC) method is also considered due to its stable and simple computation compared to other conventional MCDM methods. Therefore, an interval-valued fermatean fuzzy MABAC method is proposed and solved to select a green supplier based on multiple criteria. The proposed method's comparative analysis and sensitivity analysis validate the proposed method's effectiveness and robustness.
\end{abstract}

Keywords: Interval-valued Fermatean fuzzy; MABAC; Green supplier selection; MCDM;

Hamming distance 


\section{Introduction}

In many multi-criteria decision-making $(\mathrm{MCDM})$ problems, the preferences of experts or decision-makers are generally expressed in linguistic terms, which are quantified using a linguistic term scale such as the Likert scale. However, the Likert scale cannot handle vagueness or ambiguity of preference because it allows a single value to each linguistic term (Mathew et al., 2020a). To overcome this drawback, fuzzy set theory was introduced in 1965 (Zadeh, 1965) to capture vagueness in preference (Kahraman et al., 2016) but is unable to address the issue of uncertainty because the theory only consider the degree of satisfaction (degree of membership) and does not consider degree of dissatisfaction (degree of nonmembership) (Mathew et al., 2020b). To quantify full information, Intuitionistic Fuzzy Set (IFS) was introduced by Atanassov (1986), which accommodates both degrees of satisfaction and degree of dissatisfaction, bounded by one. Yager (2013) proposed a Pythagorean Fuzzy Set (PFS) in which the sum of the squares of the degree of satisfaction and degree of dissatisfaction is bounded by one. Later, Yager (2016) introduced a general class of similar sets and called them q-rung orthopair fuzzy sets (q-ROFS). The sum of the $q^{\text {th }}$ power of the degree of satisfaction and degree of dissatisfaction is bounded by one. Senapati and Yager (2020) proposed Fermatean Fuzzy Sets (FFS), in which the sum of the cube of the degree of satisfaction and degree of dissatisfaction is bounded by one. Compared with IFS and PFS, FFS can handle uncertainty (D. Liu et al., 2019a) as the FFS sets are larger than those of IFS and PFS. For example, if a decision-maker assesses the degree of satisfaction of an alternative as 0.9 and the degree of dissatisfaction as 0.5 in the fuzzy set, then it cannot be considered in an IFS as $0.9+0.5=1.4>1$ and PFS as $0.9^{2}+0.5^{2}=1.06>1$, but can be considered in an FFS as $0.9^{3}+0.5^{3}=0.854<1$.

Various researchers have proposed different decision-making models to solve different decision-making problems. These methods differ in their mathematical approach, and different ranking results are often obtained. Thus, the final results depend on the evaluating 
criteria and the selected decision-making model. Multi Attributive Border Approximation Area Comparison (MABAC) is a technique initially proposed by Pamučar and Ćirović (2015) in which the best alternative is selected based on the distance between each alternative and the border approximation area (BAA). Pamučar and Ćirović (2015) conducted a consistency analysis on the results obtained by the MABAC. They compared it with the results obtained from Simple Additive Weighting (SAW) Method (Fishburn, 1967), Complex Proportional Assessment (COPRAS) (Zavadskas et al., 1994), Technique for Order of Preference by Similarity to Ideal Solution (TOPSIS) (Hwang and Yoon, 1981), Multi-Objective Optimization on the basis of Ratio Analysis (MOORA) (Brauers and Zavadskas, 2006) and VlseKriterijumska Optimizacija I Kompromisno Resenje (VIKOR) (Opricovic, 1998) methods. MABAC was more stable and provided consistent solutions compared with SAW, COPRAS, TOPSIS, MOORA and VIKOR methods. Wang et al. (2020) note that MABAC is simple in computation compared with other MCDM methods and can be combined with other MCDM methods. Thus, MABAC is considered a good decision-making method to obtain reasonable results. MABAC was combined with interval type-2 fuzzy set by Yu et al. (2017) to select hotels from a tourism website to quantify complete information. MABAC was used to select a smartphone in an intuitionistic fuzzy environment by Mishra et al. (2020b). Liang et al. (2019) solved a human resource management problem using MABAC under intuitionistic fuzzy environment, using a Dombi generalized $\lambda$-Shapley Choquet arithmetical and geometric average operator to aggregate the intuitionistic fuzzy information. The concept of intuitionistic fuzzy rough numbers was proposed by Jia et al. (2019) who used intuitionistic fuzzy rough number based MABAC to solve a supplier selection problem for medical devices. Mishra et al. (2020a) and Xue et al. (2016) integrated MABAC with interval-valued intuitionistic fuzzy sets to solve programming language selection and material selection problems, respectively. The Pythagorean fuzzy set was combined with MABAC by 
Peng and Yang (2016) to solve two capital investment problems. Wang et al. (2020) used qrung orthopair fuzzy set with MABAC to solve a construction project selection problem, using Hamming distance to calculate the distance between each alternative and border approximation area. Single-valued neutrosophic MABAC and single-valued neutrosophic TOPSIS were used by Peng and Dai (2018) to solve a book publisher selection problem. It is difficult to quantify the opinion of an expert in fuzzy set theory as a number, so, to represent this degree of certainty, interval-valued fuzzy set was proposed independently by L. A. Zadeh (1975), Grattan-Guinness (1976) and Jahn (1975). Ashtiani et al. (2009) state that intervalvalued fuzzy set theory can provide more accurate modeling, so interval-valued fuzzy set theories can be used to solve real-life decision-making problems.

Supplier selection is a crucial task for any organization to be in a strategically competitive position as it directly influences the profitability and cash flow of the organization (Kumar et al., 2014). The decline in natural resources, increase in environmental pollution, and other environmental concerns have pushed companies to focus more on environmental competency. They have made environmental management an integral part of supply chain management. Thus, selecting a green supplier can improve the company's performance, profit share, and customer satisfaction (Gitinavard et al., 2018). There are various factors/criteria such as cost, quality and service, the ability of the suppliers to cope with the existing and emerging technologies, environmental and social responsibilities, carbon footprint, water usage and recycling capacity, which influence the selection of a green supplier. It is tough for decision-makers or experts to choose the best or appropriate supplier that fulfils the requirements of each criterion. Thus, the selection of green supplier is considered as a MCDM problem, as it deals with the assessment of a number of suppliers with respect to a number of criteria (Rouyendegh et al., 2020). Since the criteria are subjective in nature, they are expressed linguistically and may suffer ambiguity and 
uncertainty. To deal with this ambiguity and uncertainty, many researchers have used fuzzy set theories such as intuitionistic fuzzy set (Tian et al., 2018),(Kumari and Mishra, 2020),(Xiao et al., 2020) (Rouyendegh et al., 2020), interval-valued intuitionistic fuzzy set (Shi et al., 2018), Pythagorean fuzzy set (Fan et al., 2019), (Zhou and Chen, 2020), (Çalık, 2021), interval-valued Pythagorean fuzzy set (Wu et al., 2019), q-rung orthopair fuzzy set (R. Wang and Li, 2018), (Krishankumar et al., 2020), interval-valued q- rung orthopair fuzzy set (J. Wang et al., 2019), (L. Liu et al., 2019b).

From the aforementioned literature, it can be seen that MABAC is a simple and stable MCDM method, and researchers have combined MABAC with fuzzy set theories like interval type-2 fuzzy set, intuitionistic fuzzy set, intuitionistic fuzzy rough set, interval-valued intuitionistic fuzzy sets, Pythagorean fuzzy set and q- rung orthopair fuzzy set to quantify full information and handle ambiguity and uncertainty. The newly developed FFS is considered better than the existing IFS and PFS, whereas the interval extension of FFS can provide more accurate modeling. So in this paper, an interval extension of FFS is proposed and applied in MABAC. As the green supplier selection problem involves subjective criteria that have ambiguity and uncertainty in preferences, the proposed interval-valued fermatean fuzzy MABAC is utilized to solve a green supplier selection problem.

The main novelty and contribution of this paper are:

- An interval extension of fermatean fuzzy set is proposed with its fundamental set operations, arithmetic operations and corresponding properties.

- A Hamming distance function and score function of the interval-valued fermatean fuzzy numbers is proposed. The Hamming distance function is used to calculate the distance between each alternative and border approximation area, and the score function is used to compare each alternative and border approximation area. 
- Complete framework of interval-valued fermatean fuzzy MABAC method is proposed.

The remainder of this paper is structured as follows. Section 2 reviews the preliminaries of the fermatean fuzzy set. Section 3 introduces the proposed interval-valued fermatean fuzzy set (IVFFS), its set operations, arithmetic operations, interval-valued fermatean fuzzy Hamming distance and score function. Section 4 presents steps used in the conventional MABAC method. In section 5, we propose the MABAC using IVFFS. A numerical example (green supplier selection) is solved using the proposed interval-valued fermatean fuzzy MABAC in section 6 . In section 7 , we compare the results obtained by interval-valued fermatean fuzzy MABAC with the results obtained by previous researchers. Section 8 provides the conclusions of the work.

\section{Preliminaries}

In order to understand the mathematics of the interval-valued fermatean set, this section introduces intuitionistic fuzzy sets, Pythagorean fuzzy sets and fermatean fuzzy sets. The set operations, arithmetic operations and score function of the fermatean fuzz set are elaborated. Since we propose MABAC with an interval-valued fermatean set, MABAC is also introduced in this section. 


\subsection{Fermatean fuzzy set}

Let $\mathrm{X}$ be a universe of discourse. The membership grades of element $\mathrm{x}$ in the orthopair fuzzy sets be $\mu_{0}(x): X \in[0-1]$ and $v_{0}(x): X \in[0-1]$, where $\mu_{O}(x)$ indicates the degree of support and $v_{0}(x)$ represents the degree of support against the membership in the fuzzy set. Yager (2016) introduced a general relation between these membership grades and termed them as q-rung orthopair fuzzy set. In a q-rung orthopair fuzzy set $\left(\mu_{0}(x)\right)^{q}+\left(v_{0}(x)\right)^{q}=$ 1. The value of q represents the space of acceptable orthopairs and governs the freedom of decision-makers to express their belief about membership grades. When $\mathrm{q}=1$, $\mathrm{q}-\mathrm{ROFS}$ is IFS, for $\mathrm{q}=2$, $\mathrm{q}-\mathrm{ROFS}$ is PFS and when $\mathrm{q}=3$, $\mathrm{q}-\mathrm{ROFS}$ is FFS.

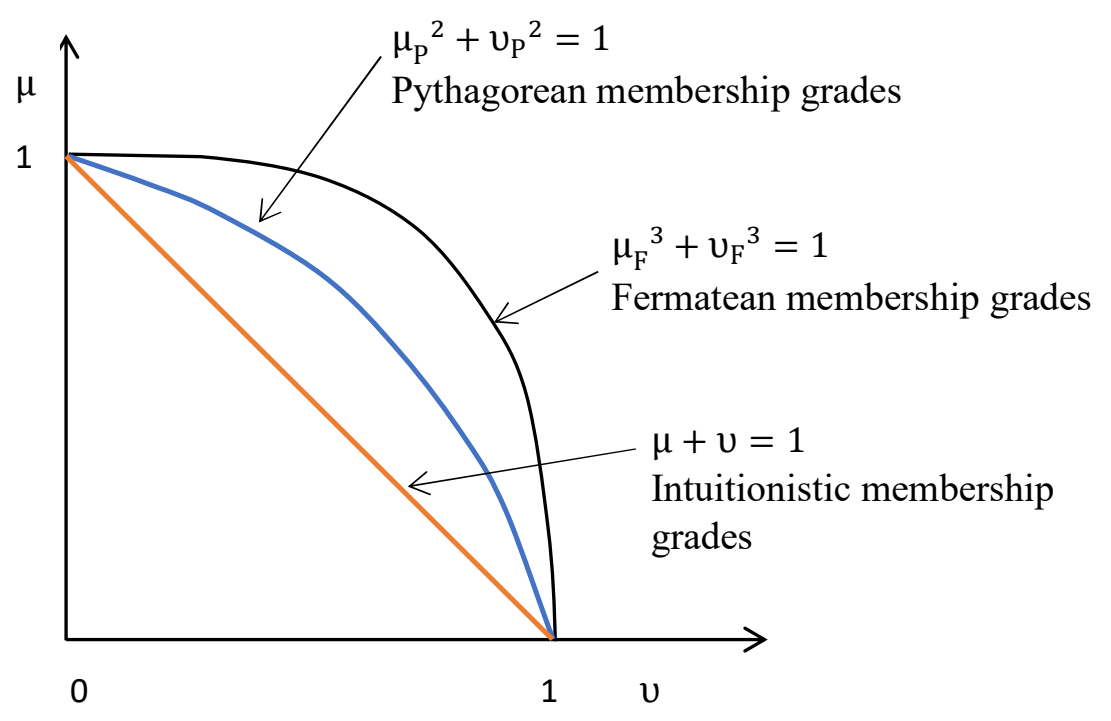

Figure 1. Space representation of Fermatean membership grades

\section{Intuitionistic fuzzy sets (Atanassov, 1986)}

An intuitionistic fuzzy set $I$ in $X$ is an object having the form $I=\left\{\left(x, \mu_{I}(x), v_{I}(x)\right): x \in X\right\}$, where $\mu_{I}(x): X \in[0-1]$ is degree of membership and $v_{I}(x): X \in[0-1]$ is degree of nonmembership of element $x$ in the set $P$ satisfying the condition, $0 \leq \mu_{I}(x)+v_{I}(x) \leq 1$.

Pythagorean fuzzy sets (Yager, 2013) 
A Pythagorean fuzzy set $P$ in $X$ is an object having the form $P=\left\{\left(x, \mu_{P}(x), v_{P}(x)\right): x \in X\right\}$, where $\mu_{P}(x): X \in[0-1]$ is degree of membership and $v_{P}(x): X \in[0-1]$ is degree of nonmembership of element $\mathrm{x}$ in the set $\mathrm{P}$ satisfying the condition, $0 \leq\left(\mu_{\mathrm{P}}(\mathrm{x})\right)^{2}+\left(\mathrm{v}_{\mathrm{P}}(\mathrm{x})\right)^{2} \leq 1$. The degree of indeterminacy of $x$ to $F$ is $\pi_{F}(x)=\left(1-\left(\mu_{F}(x)\right)^{2}-\left(v_{F}(x)\right)^{2}\right)^{\frac{1}{2}}$.

\section{Fermatean fuzzy sets (Senapati and Yager, 2020)}

A Fermatean fuzzy set $F$ in $X$ is an object having the form $F=\left\{\left(x, \mu_{F}(x), u_{F}(x)\right): x \in X\right\}$, where $\mu_{\mathrm{F}}(\mathrm{x}): \mathrm{X} \in[0-1]$ is degree of membership and $v_{\mathrm{F}}(\mathrm{x}): \mathrm{X} \in[0-1]$ is degree of nonmembership of element $\mathrm{x}$ in the set $\mathrm{F}$ satisfying the condition, $0 \leq\left(\mu_{\mathrm{F}}(\mathrm{x})\right)^{3}+\left(\mathrm{v}_{\mathrm{F}}(\mathrm{x})\right)^{3} \leq 1$. The degree of indeterminacy of $x$ to $F$ is $\pi_{F}(x)=\left(1-\left(\mu_{F}(x)\right)^{3}-\left(v_{F}(x)\right)^{3}\right)^{\frac{1}{3}}$.

Definition 2.1 (Senapati and Yager, 2020) Let $F_{1}=\left(\mu_{\mathrm{F} 1}, v_{\mathrm{F} 1}\right)$ and $F_{2}=\left(\mu_{\mathrm{F} 2}, v_{\mathrm{F} 2}\right)$ be two fermatean fuzzy numbers and $F=\left(\mu_{F}, v_{F}\right)$ be a fermatean fuzzy number then the set operations are defined as follows:

$$
\begin{array}{ll}
\text { i. } & \mathrm{F}_{1} \cup \mathrm{F}_{2}=\left(\max \left\{\mu_{\mathrm{F} 1}, \mu_{\mathrm{F} 2}\right\}, \min \left\{\mathrm{v}_{\mathrm{F} 1}, \mathrm{v}_{\mathrm{F} 2}\right\} \mathrm{H}\right) \\
\text { ii. } & \mathrm{F}_{1} \cap \mathrm{F}_{2}=\left(\min \left\{\mu_{\mathrm{F} 1}, \mu_{\mathrm{F} 2}\right\}, \max \left\{\mathrm{v}_{\mathrm{F} 1}, \mathrm{v}_{\mathrm{F} 2}\right\}\right) \\
\text { iii. } & \mathrm{F}^{c}=\left(\mathrm{v}_{\mathrm{F}}, \mu_{\mathrm{F}}\right)
\end{array}
$$

Definition 2.2 (Senapati and Yager, 2019, 2020) Let $F_{1}=\left(\mu_{\mathrm{F} 1}, \mathrm{v}_{\mathrm{F} 1}\right)$ and $\mathrm{F}_{2}=\left(\mu_{\mathrm{F} 2}, \mathrm{v}_{\mathrm{F} 2}\right)$ be two fermatean fuzzy numbers and $F=\left(\mu_{F}, v_{F}\right)$ be a fermatean fuzzy number then the arithmetic operations are defined as follows:

$$
\begin{array}{ll}
\text { i. } & \mathrm{F}_{1} \oplus \mathrm{F}_{2}=\left(\sqrt[3]{\mu_{\mathrm{F} 1}^{3}+\mu_{\mathrm{F} 2}^{3}-\mu_{\mathrm{F} 1}^{3} \times \mu_{\mathrm{F} 2}{ }^{3}}, \mathrm{v}_{\mathrm{F} 1} \times \mathrm{v}_{\mathrm{F} 2}\right) \\
\text { ii. } & \mathrm{F}_{1} \ominus \mathrm{F}_{2}=\left(\sqrt[3]{\frac{\mu_{\mathrm{F} 1}{ }^{3}-\mu_{\mathrm{F} 2}}{1-\mu_{\mathrm{F} 2}}}, \frac{v_{\mathrm{F} 1}}{v_{\mathrm{F} 2}}\right) \text { if } \mu_{\mathrm{F} 1} \geq \mu_{\mathrm{F} 2}, \mathrm{v}_{\mathrm{F} 1} \leq\left(v_{\mathrm{F} 2}, \frac{v_{\mathrm{F} 1} \times \pi_{\mathrm{F} 1}}{\pi_{\mathrm{F} 2}}\right) \\
\text { iii. } & \mathrm{F}_{1} \otimes \mathrm{F}_{2}=\left(\mu_{\mathrm{F} 1} \times \mu_{\mathrm{F} 2}, \sqrt[3]{\mathrm{v}_{\mathrm{F} 1}^{3}+\mathrm{v}_{\mathrm{F} 2}{ }^{3}-\mathrm{v}_{\mathrm{F} 1}{ }^{3} \times \mathrm{v}_{\mathrm{F} 2}^{3}}\right)
\end{array}
$$


iv. $\quad F_{1} \oslash F_{2}=\left(\frac{\mu_{F 1}}{\mu_{F 2}}, \sqrt[3]{\frac{v_{F 1}{ }^{3}-v_{F 2}}{1-v_{F 2}^{3}}}\right)$ if $\mu_{\mathrm{F} 1} \leq\left(\mu_{F 2}, \frac{\mu_{F 1} \times \pi_{F 1}}{\pi_{F 2}}\right), v_{F 1} \geq v_{F 2}$

v. $\mathrm{kF}=\left(\sqrt[3]{1-\left(1-\mu_{\mathrm{F}}^{3}\right)^{\mathrm{k}}}, \mathrm{v}_{\mathrm{F}}^{k}\right)$, where $\mathrm{k}>0$

vi. $\quad \mathrm{F}^{\mathrm{k}}=\left(\mu_{\mathrm{F}}^{k}, \sqrt[3]{1-\left(1-\mathrm{v}_{\mathrm{F}}^{3}\right)^{\mathrm{k}}}\right)$, where $\mathrm{k}>0$

Definition 2.3 (Senapati and Yager, 2020) For any fermatean fuzzy number $F=\left(\mu_{F}, v_{F}\right)$, the suggested score function score $(F) \in[-1,1]$ score $(F)=\mu_{F}^{3}-v_{F}^{3}$

\subsection{MABAC}

The steps in conventional MABAC method (Pamučar and Ćirović, 2015) are described below:

Step-1: Construction of initial decision matrix. The alternatives are evaluated with respect to different criteria. $x_{i j}$ is the performance value of $i^{\text {th }}$ alternative $(i=1,2, \ldots, m)$ with respect to $\mathrm{j}^{\text {th }}$ criteria $(\mathrm{j}=1,2, \ldots, \mathrm{n})$.

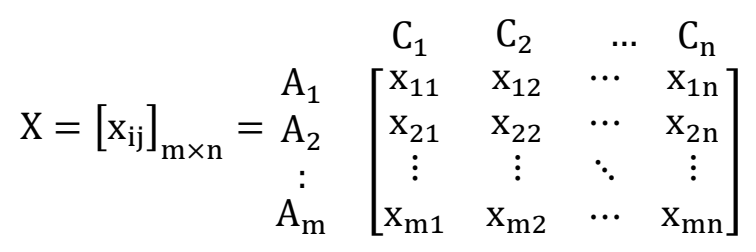

Where $\mathrm{m}$ is the number of alternatives and $\mathrm{n}$ is the number of criteria.

Step-2: Normalize the initial decision matrix. The normalized matrix $\mathrm{N}$ is computed by normalizing the performance value $\mathrm{x}_{\mathrm{ij}}$.

$$
\mathrm{N}=\left[\mathrm{n}_{\mathrm{ij}}\right]_{\mathrm{m} \times \mathrm{n}}=\begin{array}{ccccc} 
& \mathrm{C}_{1} & \mathrm{C}_{2} & \cdots & \mathrm{C}_{\mathrm{n}} \\
& \mathrm{A}_{1} \\
\mathrm{~A}_{2} & \vdots \\
\mathrm{A}_{\mathrm{m}}
\end{array}\left[\begin{array}{cccc}
\mathrm{n}_{11} & \mathrm{n}_{12} & \cdots & \mathrm{n}_{1 \mathrm{n}} \\
\mathrm{n}_{21} & \mathrm{n}_{22} & \cdots & \mathrm{n}_{2 \mathrm{n}} \\
\vdots & \vdots & \ddots & \vdots \\
\mathrm{n}_{\mathrm{m} 1} & \mathrm{n}_{\mathrm{m} 2} & \cdots & \mathrm{n}_{\mathrm{mn}}
\end{array}\right]
$$

The performance values are normalized using the below equation:

$\mathrm{n}_{\mathrm{ij}}=\frac{\mathrm{x}_{\mathrm{ij}}-x_{i}^{-}}{x_{i}^{+}-x_{i}^{-}} \quad$ for beneficial criteria.

$\mathrm{n}_{\mathrm{ij}}=\frac{\mathrm{x}_{\mathrm{ij}}-x_{i}^{+}}{x_{i}^{-}-x_{i}^{+}} \quad$ for non-beneficial criteria. 
Where $x_{i}^{+}=\max \left(\mathrm{x}_{\mathrm{i} 1}, \mathrm{x}_{\mathrm{i} 2}, \ldots, \mathrm{x}_{\mathrm{im}}\right)$ and $x_{i}^{-}=\max \left(\mathrm{x}_{\mathrm{i} 1}, \mathrm{x}_{\mathrm{i} 2}, \ldots, \mathrm{x}_{\mathrm{im}}\right)$

Step-3: Compute the weighted normalized matrix. The weighted normalized values are computed using the equation:

$$
\begin{aligned}
& \mathrm{v}_{\mathrm{ij}}=\mathrm{w}_{\mathrm{j}} \cdot\left(\mathrm{n}_{\mathrm{ij}}+1\right)
\end{aligned}
$$

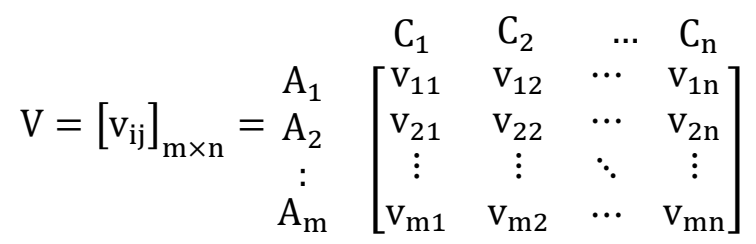

$$
\begin{aligned}
& \mathrm{C}_{1} \quad \mathrm{C}_{2} \quad \ldots \quad \mathrm{C}_{\mathrm{n}} \\
& \mathrm{A}_{1} \quad\left[\begin{array}{llll}
\mathrm{w}_{1} \cdot\left(\mathrm{n}_{11}+1\right) & \mathrm{w}_{2} \cdot\left(\mathrm{n}_{12}+1\right) & \cdots & \mathrm{w}_{\mathrm{n}} \cdot\left(\mathrm{n}_{1 \mathrm{n}}+1\right)
\end{array}\right] \\
& \begin{array}{c|cccc}
=A_{2} & w_{1} \cdot\left(n_{21}+1\right) & w_{2} \cdot\left(n_{22}+1\right) & \cdots & w_{n} \cdot\left(n_{2 n}+1\right) \\
\vdots & \vdots & & \ddots &
\end{array}
\end{aligned}
$$

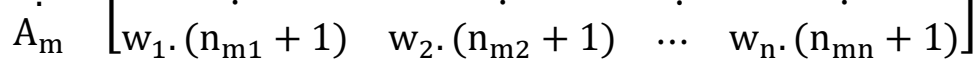

Step-4: Compute the border approximation area (BAA) matrix $(\mathrm{G})$ for each criterion.

$$
\begin{aligned}
& g_{j}=\left(\prod_{i=1}^{m} v_{i j}\right)^{1 / m} \\
& \left.G=\left[g_{j}\right]_{1 \times n}=\begin{array}{llll}
C_{1} & C_{2} & \ldots & C_{n} \\
g_{1} & g_{2} & \ldots & g_{n}
\end{array}\right]
\end{aligned}
$$

Step-5: Compute the distance of the alternative from the BAA. The distance of the alternatives from the BAA is computed by taking the difference between the elements in the weighted matrix (V) and the value of BAA.

$$
\begin{aligned}
& \mathrm{D}=\left[\mathrm{d}_{\mathrm{ij}}\right]_{\mathrm{m} \times \mathrm{n}}=\begin{array}{c}
\mathrm{C}_{1} \\
\mathrm{~A}_{1} \\
\mathrm{~A}_{2} \\
\vdots \\
\mathrm{A}_{\mathrm{m}}
\end{array}\left[\begin{array}{cccc}
\mathrm{d}_{11} & \mathrm{~d}_{12} & \cdots & \mathrm{C}_{\mathrm{n}} \\
\mathrm{d}_{21} & \mathrm{~d}_{22} & \cdots & \mathrm{d}_{2 \mathrm{n}} \\
\vdots & \vdots & \ddots & \vdots \\
\mathrm{d}_{\mathrm{m} 1} & \mathrm{~d}_{\mathrm{m} 2} & \cdots & \mathrm{d}_{\mathrm{mn}}
\end{array}\right] \\
& \begin{array}{c} 
\\
\mathrm{A}_{1} \\
\mathrm{~A}_{2} \\
\vdots \\
\mathrm{A}_{\mathrm{m}}
\end{array}\left[\begin{array}{cccc}
\mathrm{v}_{11}-\mathrm{g}_{1} & \mathrm{v}_{12}-\mathrm{g}_{2} & \cdots & \mathrm{v}_{1 \mathrm{n}}-\mathrm{g}_{\mathrm{n}} \\
\mathrm{v}_{21}-\mathrm{g}_{1} & \mathrm{v}_{22}-\mathrm{g}_{2} & \cdots & \mathrm{v}_{2 \mathrm{n}}-\mathrm{g}_{\mathrm{n}} \\
\vdots & \vdots & \ddots & \vdots \\
\mathrm{v}_{\mathrm{m} 1}-\mathrm{g}_{1} & \mathrm{v}_{\mathrm{m} 2-\mathrm{g}_{2}} & \cdots & \mathrm{v}_{\mathrm{mn}}-\mathrm{g}_{\mathrm{n}}
\end{array}\right]
\end{aligned}
$$


Step-6: Ranking the alternatives. The alternatives are ranked based on the comprehensive evaluation result $\left(S_{i}\right)$ which is computed by summing the distance values of each alternative. The larger the value of $S_{i}$, the better is the alternative.

$$
\mathrm{S}_{\mathrm{i}}=\sum_{j=1}^{n} \mathrm{~d}_{\mathrm{ij}}
$$

\section{The Proposed Interval-valued Fermatean Fuzzy Sets}

An Interval-valued Fermatean fuzzy set $\tilde{F}$ in $X$ is an object having the form $\tilde{F}=$ $\left\{\left(x,\left[\mu_{\widetilde{F}}^{l}(x), \mu_{\widetilde{F}}^{u}(x)\right],\left[v_{\widetilde{F}}^{l}(x), v_{\widetilde{F}}^{u}(x)\right]\right): x \in X\right\}$, where $\left[\mu_{\widetilde{F}}^{l}(x), \mu_{\widetilde{F}}^{u}(x)\right]: X \in[0-1]$ is degree of membership and $\left[v_{\widetilde{F}}^{l}(x), v_{\widetilde{F}}^{u}(x)\right]: X \in[0-1]$ is degree of non-membership of element $x$ in the set $\tilde{\mathrm{F}}$ satisfying the condition, $0 \leq\left(\mu_{\widetilde{\mathrm{F}}}^{\mathrm{u}}(\mathrm{x})\right)^{3}+\left(\mathrm{v}_{\widetilde{\mathrm{F}}}^{\mathrm{u}}(\mathrm{x})\right)^{3} \leq 1$. The degree of indeterminacy of $\mathrm{x}$ to $\tilde{\mathrm{F}}$ is $\left[\pi_{\widetilde{\mathrm{F}}}^{1}(\mathrm{x}), \pi_{\widetilde{\mathrm{F}}}^{\mathrm{u}}(\mathrm{x})\right]=\left[\left(1-\left(\mu_{\widetilde{\mathrm{F}}}^{\mathrm{u}}(\mathrm{x})\right)^{3}-\left(\mathrm{v}_{\widetilde{\mathrm{F}}}^{\mathrm{u}}(\mathrm{x})\right)^{3}\right)^{\frac{1}{3}},\left(1-\left(\mu_{\widetilde{\mathrm{F}}}^{1}(\mathrm{x})\right)^{3}-\left(\mathrm{v}_{\widetilde{\mathrm{F}}}^{\mathrm{l}}(\mathrm{x})\right)^{3}\right)^{\frac{1}{3}}\right]$. For convenience, we call $\widetilde{F}=\left(\left[\mu_{\widetilde{F}}^{l}, \mu_{\widetilde{F}}^{u}\right],\left[v_{\widetilde{F}}^{l}, v_{\widetilde{F}}^{u}\right]\right)$ an Interval-valued Fermatean Fuzzy Number (IVFFN).

Definition 3.1 Let $\widetilde{F}_{1}=\left(\left[\mu_{1}^{\mathrm{l}}, \mu_{1}^{\mathrm{u}}\right],\left[v_{1}^{\mathrm{l}}, \mathrm{v}_{1}^{\mathrm{u}}\right]\right)$ and $\widetilde{\mathrm{F}}_{2}=\left(\left[\mu_{2}^{\mathrm{l}}, \mu_{2}^{\mathrm{u}}\right],\left[v_{2}^{\mathrm{l}}, \mathrm{v}_{2}^{\mathrm{u}}\right]\right)$ be two IVFFNs, then:

$\tilde{\mathrm{F}}_{1}=\tilde{\mathrm{F}}_{2}$ iff $\mu_{1}^{\mathrm{l}}=\mu_{2}^{\mathrm{l}}, \mu_{1}^{\mathrm{u}}=\mu_{2}^{\mathrm{u}}, v_{1}^{\mathrm{l}}=v_{2}^{l}$ and $v_{1}^{\mathrm{u}}=v_{2}^{\mathrm{u}}$

$\tilde{\mathrm{F}}_{1}<\tilde{\mathrm{F}}_{2}$ iff $\mu_{1}^{\mathrm{l}} \leq \mu_{2}^{\mathrm{l}}, \mu_{1}^{\mathrm{u}} \leq \mu_{2}^{\mathrm{u}}, v_{1}^{\mathrm{l}} \geq v_{2}^{l}$ and $v_{1}^{\mathrm{u}} \geq v_{2}^{\mathrm{u}}$

Definition 3.2 Let $\tilde{\mathrm{F}}=\left(\left[\mu^{\mathrm{l}}, \mu^{\mathrm{u}}\right],\left[\mathrm{v}^{\mathrm{l}}, \mathrm{v}^{\mathrm{u}}\right]\right), \quad \tilde{\mathrm{F}}_{1}=\left(\left[\mu_{1}^{\mathrm{l}}, \mu_{1}^{\mathrm{u}}\right],\left[\mathrm{v}_{1}^{\mathrm{l}}, \mathrm{v}_{1}^{\mathrm{u}}\right]\right)$ and $\tilde{\mathrm{F}}_{2}=$ $\left(\left[\mu_{2}^{\mathrm{l}}, \mu_{2}^{\mathrm{u}}\right],\left[v_{2}^{\mathrm{l}}, \mathrm{v}_{2}^{\mathrm{u}}\right]\right)$ be three IVFFNs, then the set operations are defined as follows:

$$
\begin{array}{ll}
\text { i. } & \tilde{\mathrm{F}}_{1} \cup \tilde{\mathrm{F}}_{2}=\left(\left[\max \left\{\mu_{1}^{\mathrm{l}}, \mu_{2}^{\mathrm{l}}\right\}, \max \left\{\mu_{1}^{\mathrm{u}}, \mu_{2}^{u}\right\}\right],\left[\min \left\{\mathrm{v}_{1}^{\mathrm{l}}, \mathrm{v}_{2}^{\mathrm{l}}\right\}, \min \left\{\mathrm{v}_{1}^{\mathrm{u}}, \mathrm{v}_{2}^{u}\right\}\right]\right) \\
\text { ii. } & \tilde{\mathrm{F}}_{1} \cap \tilde{\mathrm{F}}_{2}=\left(\left[\min \left\{\mu_{1}^{\mathrm{l}}, \mu_{2}^{\mathrm{l}}\right\}, \min \left\{\mu_{1}^{\mathrm{u}}, \mu_{2}^{u}\right\}\right],\left[\max \left\{\mathrm{v}_{1}^{\mathrm{l}}, \mathrm{v}_{2}^{\mathrm{l}}\right\}, \max \left\{\mathrm{v}_{1}^{\mathrm{u}}, \mathrm{v}_{2}^{u}\right\}\right]\right) \\
\text { iii. } & \tilde{\mathrm{F}}^{\mathrm{c}}=\left(\left[\mathrm{v}^{\mathrm{l}}, \mathrm{v}^{\mathrm{u}}\right],\left[\mu^{\mathrm{l}}, \mu^{\mathrm{u}}\right]\right)
\end{array}
$$


Theorem Let $\tilde{\mathrm{F}}_{1}=\left(\left[\mu_{1}^{\mathrm{l}}, \mu_{1}^{\mathrm{u}}\right],\left[\mathrm{v}_{1}^{\mathrm{l}}, \mathrm{v}_{1}^{\mathrm{u}}\right]\right) \quad, \quad \tilde{\mathrm{F}}_{2}=\left(\left[\mu_{2}^{\mathrm{l}}, \mu_{2}^{\mathrm{u}}\right],\left[\mathrm{v}_{2}^{\mathrm{l}}, \mathrm{v}_{2}^{\mathrm{u}}\right]\right)$ and $\tilde{\mathrm{F}}_{3}=$ $\left(\left[\mu_{3}^{\mathrm{l}}, \mu_{3}^{\mathrm{u}}\right],\left[v_{3}^{\mathrm{l}}, \mathrm{v}_{3}^{\mathrm{u}}\right]\right)$ be three IVFFNs, then the following are valid for intersection and union of IVFFNs.

Commutative property:
i. $\quad \tilde{\mathrm{F}}_{1} \cup \tilde{\mathrm{F}}_{2}=\tilde{\mathrm{F}}_{2} \cup \tilde{\mathrm{F}}_{1}$
ii. $\quad \tilde{\mathrm{F}}_{1} \cap \tilde{\mathrm{F}}_{2}=\tilde{\mathrm{F}}_{2} \cap \tilde{\mathrm{F}}_{1}$

Associative property:
iii. $\quad \tilde{\mathrm{F}}_{1} \cup\left(\widetilde{\mathrm{F}}_{2} \cup \tilde{\mathrm{F}}_{3}\right)=\left(\tilde{\mathrm{F}}_{1} \cup \tilde{\mathrm{F}}_{2}\right) \cup \tilde{\mathrm{F}}_{3}$
iv. $\quad \tilde{\mathrm{F}}_{1} \cap\left(\tilde{\mathrm{F}}_{2} \cap \tilde{\mathrm{F}}_{3}\right)=\left(\tilde{\mathrm{F}}_{1} \cap \tilde{\mathrm{F}}_{2}\right) \cap \tilde{\mathrm{F}}_{3}$

De Morgan's laws"
v. $\left(\tilde{\mathrm{F}}_{1} \cup \tilde{\mathrm{F}}_{2}\right)^{\mathrm{c}}=\tilde{\mathrm{F}}_{1}{ }^{\mathrm{c}} \cap \tilde{\mathrm{F}}_{2}{ }^{\mathrm{c}}$
vi. $\quad\left(\tilde{\mathrm{F}}_{1} \cap \tilde{\mathrm{F}}_{2}\right)^{\mathrm{c}}=\tilde{\mathrm{F}}_{1}{ }^{\mathrm{c}} \mathrm{U} \tilde{\mathrm{F}}_{2}{ }^{\mathrm{c}}$

Proof According to definition 3.2 we can obtain:

$$
\begin{aligned}
& \text { i. } \quad \tilde{\mathrm{F}}_{1} \cup \tilde{\mathrm{F}}_{2} \\
= & \left(\left[\max \left\{\mu_{1}^{\mathrm{l}}, \mu_{2}^{\mathrm{l}}\right\}, \max \left\{\mu_{1}^{\mathrm{u}}, \mu_{2}^{u}\right\}\right],\left[\min \left\{\mathrm{v}_{1}^{\mathrm{l}}, \mathrm{v}_{2}^{\mathrm{l}}\right\}, \min \left\{\mathrm{v}_{1}^{\mathrm{u}}, \mathrm{v}_{2}^{u}\right\}\right]\right) \\
= & \left(\left[\max \left\{\mu_{2}^{\mathrm{l}}, \mu_{1}^{\mathrm{l}}\right\}, \max \left\{\mu_{2}^{\mathrm{u}}, \mu_{1}^{u}\right\}\right],\left[\min \left\{\mathrm{v}_{2}^{\mathrm{l}}, \mathrm{v}_{1}^{\mathrm{l}}\right\}, \min \left\{\mathrm{v}_{2}^{\mathrm{u}}, \mathrm{v}_{1}^{u}\right\}\right]\right) \\
= & \tilde{\mathrm{F}}_{2} \cup \tilde{\mathrm{F}}_{1} \\
& \text { ii. } \quad \tilde{\mathrm{F}}_{1} \cap \tilde{\mathrm{F}}_{2} \\
= & \left(\left[\min \left\{\mu_{1}^{\mathrm{l}}, \mu_{2}^{\mathrm{l}}\right\}, \min \left\{\mu_{1}^{\mathrm{u}}, \mu_{2}^{u}\right\}\right],\left[\max \left\{\mathrm{v}_{1}^{\mathrm{l}}, \mathrm{v}_{2}^{\mathrm{l}}\right\}, \max \left\{\mathrm{v}_{1}^{\mathrm{u}}, \mathrm{v}_{2}^{u}\right\}\right]\right) \\
= & \left(\left[\min \left\{\mu_{2}^{\mathrm{l}}, \mu_{1}^{\mathrm{l}}\right\}, \min \left\{\mu_{2}^{\mathrm{u}}, \mu_{1}^{u}\right\}\right],\left[\max \left\{v_{2}^{\mathrm{l}}, \mathrm{v}_{1}^{\mathrm{l}}\right\}, \max \left\{\mathrm{v}_{2}^{\mathrm{u}}, \mathrm{v}_{1}^{u}\right\}\right]\right) \\
= & \tilde{\mathrm{F}}_{2} \cap \tilde{\mathrm{F}}_{1}
\end{aligned}
$$

iii. $\quad \tilde{\mathrm{F}}_{1} \cup\left(\widetilde{\mathrm{F}}_{2} \cup \widetilde{\mathrm{F}}_{3}\right)$ 
$=\left(\left[\mu_{1}^{\mathrm{l}}, \mu_{1}^{\mathrm{u}}\right],\left[v_{1}^{\mathrm{l}}, \mathrm{v}_{1}^{\mathrm{u}}\right]\right) \cup\left(\left[\max \left\{\mu_{2}^{\mathrm{l}}, \mu_{3}^{\mathrm{l}}\right\}, \max \left\{\mu_{2}^{\mathrm{u}}, \mu_{3}^{u}\right\}\right],\left[\min \left\{\mathrm{v}_{2}^{\mathrm{l}}, \mathrm{v}_{3}^{\mathrm{l}}\right\}, \min \left\{\mathrm{v}_{2}^{\mathrm{u}}, \mathrm{v}_{3}^{u}\right\}\right]\right)$

$=\left(\left[\max \left\{\mu_{1}^{1}, \max \left\{\mu_{2}^{1}, \mu_{3}^{1}\right\}\right\}, \max \left\{\mu_{1}^{\mathrm{u}}, \max \left\{\mu_{2}^{\mathrm{u}}, \mu_{3}^{u}\right\}\right\}\right],\left[\min \left\{\mathrm{v}_{1}^{\mathrm{l}}, \min \left\{\mathrm{u}_{2}^{\mathrm{l}}, \mathrm{v}_{3}^{\mathrm{l}}\right\}\right\}, \min \left\{\mathrm{v}_{1}^{\mathrm{u}}, \min \left\{\mathrm{v}_{2}^{\mathrm{u}}, \mathrm{v}_{3}^{u}\right\}\right\}\right]\right)$

$=\left(\left[\max \left\{\mu_{1}^{1}, \mu_{2}^{1}, \mu_{3}^{1}\right\}, \max \left\{\mu_{1}^{\mathrm{u}}, \mu_{2}^{\mathrm{u}}, \mu_{3}^{u}\right\}\right],\left[\min \left\{\mathrm{v}_{1}^{1}, v_{2}^{1}, v_{3}^{1}\right\}, \min \left\{\mathrm{v}_{1}^{\mathrm{u}}, \mathrm{v}_{2}^{\mathrm{u}}, \mathrm{v}_{3}^{u}\right\}\right]\right)$

$\left(\tilde{\mathrm{F}}_{1} \cup \tilde{\mathrm{F}}_{2}\right) \cup \tilde{\mathrm{F}}_{3}$

$=\left(\left[\max \left\{\mu_{1}^{1}, \mu_{2}^{1}\right\}, \max \left\{\mu_{1}^{\mathrm{u}}, \mu_{2}^{u}\right\}\right],\left[\min \left\{v_{1}^{1}, v_{2}^{1}\right\}, \min \left\{v_{1}^{\mathrm{u}}, v_{2}^{u}\right\}\right]\right) \cup\left(\left[\mu_{3}^{1}, \mu_{3}^{\mathrm{u}}\right],\left[\mathrm{v}_{3}^{1}, \mathrm{v}_{3}^{\mathrm{u}}\right]\right)$

$=\left(\left[\max \left\{\max \left\{\mu_{1}^{1}, \mu_{2}^{1}\right\}, \mu_{3}^{1}\right\}, \max \left\{\max \left\{\mu_{1}^{\mathrm{u}}, \mu_{2}^{u}\right\}, \mu_{3}^{\mathrm{u}}\right\}\right],\left[\min \left\{\min \left\{\mathrm{v}_{1}^{\mathrm{l}}, \mathrm{v}_{2}^{\mathrm{l}}\right\}, \mathrm{v}_{3}^{1}\right\}, \min \left\{\min \left\{\mathrm{v}_{1}^{\mathrm{u}}, \mathrm{v}_{2}^{u}\right\}, \mathrm{v}_{3}^{\mathrm{u}}\right\}\right]\right)$

$=\left(\left[\max \left\{\mu_{1}^{1}, \mu_{2}^{1}, \mu_{3}^{1}\right\}, \max \left\{\mu_{1}^{\mathrm{u}}, \mu_{2}^{\mathrm{u}}, \mu_{3}^{u}\right\}\right],\left[\min \left\{\mathrm{u}_{1}^{1}, v_{2}^{1}, v_{3}^{1}\right\}, \min \left\{\mathrm{v}_{1}^{\mathrm{u}}, \mathrm{v}_{2}^{\mathrm{u}}, \mathrm{v}_{3}^{u}\right\}\right]\right)$

Thus

$\tilde{\mathrm{F}}_{1} \cup\left(\tilde{\mathrm{F}}_{2} \cup \tilde{\mathrm{F}}_{3}\right)=\left(\tilde{\mathrm{F}}_{1} \cup \tilde{\mathrm{F}}_{2}\right) \cup \tilde{\mathrm{F}}_{3}$

iv. $\quad \tilde{\mathrm{F}}_{1} \cap\left(\tilde{\mathrm{F}}_{2} \cap \tilde{\mathrm{F}}_{3}\right)=\left(\tilde{\mathrm{F}}_{1} \cap \tilde{\mathrm{F}}_{2}\right) \cap \tilde{\mathrm{F}}_{3}$

$=\left(\left[\mu_{1}^{1}, \mu_{1}^{\mathrm{u}}\right],\left[v_{1}^{\mathrm{l}}, \mathrm{v}_{1}^{\mathrm{u}}\right]\right) \cup\left(\left[\min \left\{\mu_{2}^{\mathrm{l}}, \mu_{3}^{\mathrm{l}}\right\}, \min \left\{\mu_{2}^{\mathrm{u}}, \mu_{3}^{u}\right\}\right],\left[\max \left\{\mathrm{v}_{2}^{\mathrm{l}}, \mathrm{v}_{3}^{\mathrm{l}}\right\}, \max \left\{\mathrm{v}_{2}^{\mathrm{u}}, \mathrm{v}_{3}^{u}\right\}\right]\right)$

$=\left(\left[\min \left\{\mu_{1}^{1}, \min \left\{\mu_{2}^{1}, \mu_{3}^{1}\right\}\right\}, \min \left\{\mu_{1}^{\mathrm{u}}, \min \left\{\mu_{2}^{\mathrm{u}}, \mu_{3}^{u}\right\}\right\}\right],\left[\max \left\{v_{1}^{\mathrm{l}}, \max \left\{v_{2}^{1}, v_{3}^{\mathrm{l}}\right\}\right\}, \max \left\{v_{1}^{\mathrm{u}}, \max \left\{v_{2}^{\mathrm{u}}, v_{3}^{u}\right\}\right\}\right]\right)$

$=\left(\left[\min \left\{\mu_{1}^{1}, \mu_{2}^{1}, \mu_{3}^{\mathrm{l}}\right\}, \min \left\{\mu_{1}^{\mathrm{u}}, \mu_{2}^{\mathrm{u}}, \mu_{3}^{u}\right\}\right],\left[\max \left\{\mathrm{v}_{1}^{\mathrm{l}}, \mathrm{v}_{2}^{\mathrm{l}}, \mathrm{v}_{3}^{\mathrm{l}}\right\}, \max \left\{\mathrm{v}_{1}^{\mathrm{u}}, \mathrm{v}_{2}^{\mathrm{u}}, \mathrm{v}_{3}^{u}\right\}\right]\right)$

$\left(\tilde{\mathrm{F}}_{1} \cap \tilde{\mathrm{F}}_{2}\right) \cap \tilde{\mathrm{F}}_{3}$

$=\left(\left[\min \left\{\mu_{1}^{1}, \mu_{2}^{1}\right\}, \min \left\{\mu_{1}^{\mathrm{u}}, \mu_{2}^{u}\right\}\right],\left[\max \left\{v_{1}^{1}, v_{2}^{1}\right\}, \max \left\{v_{1}^{\mathrm{u}}, v_{2}^{u}\right\}\right]\right) \cup\left(\left[\mu_{3}^{1}, \mu_{3}^{\mathrm{u}}\right],\left[v_{3}^{1}, v_{3}^{\mathrm{u}}\right]\right)$

$=\left(\left[\min \left\{\min \left\{\mu_{1}^{1}, \mu_{2}^{1}\right\}, \mu_{3}^{1}\right\}, \min \left\{\min \left\{\mu_{1}^{\mathrm{u}}, \mu_{2}^{u}\right\}, \mu_{3}^{\mathrm{u}}\right\}\right],\left[\max \left\{\max \left\{v_{1}^{1}, v_{2}^{1}\right\}, v_{3}^{1}\right\}, \max \left\{\max \left\{v_{1}^{\mathrm{u}}, v_{2}^{u}\right\}, v_{3}^{\mathrm{u}}\right\}\right]\right)$

$=\left(\left[\min \left\{\mu_{1}^{\mathrm{l}}, \mu_{2}^{\mathrm{l}}, \mu_{3}^{\mathrm{l}}\right\}, \min \left\{\mu_{1}^{\mathrm{u}}, \mu_{2}^{\mathrm{u}}, \mu_{3}^{u}\right\}\right],\left[\max \left\{\mathrm{v}_{1}^{\mathrm{l}}, \mathrm{v}_{2}^{\mathrm{l}}, \mathrm{v}_{3}^{\mathrm{l}}\right\}, \max \left\{\mathrm{v}_{1}^{\mathrm{u}}, \mathrm{v}_{2}^{\mathrm{u}}, \mathrm{v}_{3}^{u}\right\}\right]\right)$

Thus

$\tilde{\mathrm{F}}_{1} \cap\left(\tilde{\mathrm{F}}_{2} \cap \tilde{\mathrm{F}}_{3}\right)=\left(\tilde{\mathrm{F}}_{1} \cap \tilde{\mathrm{F}}_{2}\right) \cap \tilde{\mathrm{F}}_{3}$

v. $\left(\tilde{\mathrm{F}}_{1} \cup \tilde{\mathrm{F}}_{2}\right)^{\mathrm{c}}=\tilde{\mathrm{F}}_{1}^{\mathrm{c}} \cap \tilde{\mathrm{F}}_{2}{ }^{\mathrm{c}}$

$\left(\tilde{\mathrm{F}}_{1} \cup \tilde{\mathrm{F}}_{2}\right)^{\mathrm{c}}=\left(\left(\left[\mu_{1}^{\mathrm{l}}, \mu_{1}^{\mathrm{u}}\right],\left[\mathrm{v}_{1}^{\mathrm{l}}, \mathrm{v}_{1}^{\mathrm{u}}\right]\right) \cup\left(\left[\mu_{2}^{\mathrm{l}}, \mu_{2}^{\mathrm{u}}\right],\left[v_{2}^{\mathrm{l}}, \mathrm{v}_{2}^{\mathrm{u}}\right]\right)\right)^{\mathrm{c}}$ 


$$
\begin{aligned}
& =\left(\left[\max \left\{\mu_{1}^{1}, \mu_{2}^{1}\right\}, \max \left\{\mu_{1}^{\mathrm{u}}, \mu_{2}^{u}\right\}\right],\left[\min \left\{\mathrm{v}_{1}^{\mathrm{l}}, \mathrm{v}_{2}^{\mathrm{l}}\right\}, \min \left\{\mathrm{v}_{1}^{\mathrm{u}}, \mathrm{v}_{2}^{u}\right\}\right]\right)^{\mathrm{c}} \\
& =\left(\left[\min \left\{\mathrm{v}_{1}^{\mathrm{l}}, \mathrm{v}_{2}^{\mathrm{l}}\right\}, \min \left\{\mathrm{v}_{1}^{\mathrm{u}}, \mathrm{v}_{2}^{\mathrm{u}}\right\}\right],\left[\max \left\{\mu_{1}^{\mathrm{l}}, \mu_{2}^{\mathrm{l}}\right\}, \max \left\{\mu_{1}^{\mathrm{u}}, \mu_{2}^{u}\right\}\right]\right) \\
& \tilde{\mathrm{F}}_{1}^{\mathrm{c}} \cap \tilde{\mathrm{F}}_{2}^{\mathrm{c}}=\left(\left[\mu_{1}^{\mathrm{l}}, \mu_{1}^{\mathrm{u}}\right],\left[\mathrm{v}_{1}^{\mathrm{l}}, \mathrm{v}_{1}^{\mathrm{u}}\right]\right)^{\mathrm{c}} \cap\left(\left[\mu_{2}^{1}, \mu_{2}^{\mathrm{u}}\right],\left[\mathrm{v}_{2}^{\mathrm{l}}, \mathrm{v}_{2}^{\mathrm{u}}\right]\right)^{\mathrm{c}} \\
& =\left(\left[\mathrm{v}_{1}^{\mathrm{l}}, \mathrm{v}_{1}^{\mathrm{u}}\right],\left[\mu_{1}^{\mathrm{l}}, \mu_{1}^{\mathrm{u}}\right]\right) \cap\left(\left[\mathrm{v}_{2}^{\mathrm{l}}, \mathrm{v}_{2}^{\mathrm{u}}\right],\left[\mu_{2}^{\mathrm{l}}, \mu_{2}^{\mathrm{u}}\right]\right) \\
& =\left(\left[\min \left\{\mathrm{v}_{1}^{\mathrm{l}}, \mathrm{v}_{2}^{\mathrm{l}}\right\}, \min \left\{\mathrm{v}_{1}^{\mathrm{u}}, \mathrm{v}_{2}^{\mathrm{u}}\right\}\right],\left[\max \left\{\mu_{1}^{\mathrm{l}}, \mu_{2}^{\mathrm{l}}\right\}, \max \left\{\mu_{1}^{\mathrm{u}}, \mu_{2}^{u}\right\}\right]\right)
\end{aligned}
$$

Thus

$$
\left(\tilde{\mathrm{F}}_{1} \cup \tilde{\mathrm{F}}_{2}\right)^{\mathrm{c}}=\tilde{\mathrm{F}}_{1}^{\mathrm{c}} \cap \tilde{\mathrm{F}}_{2}^{\mathrm{c}}
$$

$$
\begin{aligned}
& \text { vi. } \quad\left(\tilde{\mathrm{F}}_{1} \cap \tilde{\mathrm{F}}_{2}\right)^{\mathrm{c}}=\tilde{\mathrm{F}}_{1}{ }^{\mathrm{c}} \cup \tilde{\mathrm{F}}_{2}{ }^{\mathrm{c}} \\
& \left(\tilde{\mathrm{F}}_{1} \cap \tilde{\mathrm{F}}_{2}\right)^{\mathrm{c}}=\left(\left(\left[\mu_{1}^{\mathrm{l}}, \mu_{1}^{\mathrm{u}}\right],\left[\mathrm{v}_{1}^{\mathrm{l}}, \mathrm{v}_{1}^{\mathrm{u}}\right]\right) \cap\left(\left[\mu_{2}^{\mathrm{l}}, \mu_{2}^{\mathrm{u}}\right],\left[\mathrm{v}_{2}^{\mathrm{l}}, \mathrm{v}_{2}^{\mathrm{u}}\right]\right)\right) \\
& =\left(\left[\min \left\{\mu_{1}^{1}, \mu_{2}^{1}\right\}, \min \left\{\mu_{1}^{\mathrm{u}}, \mu_{2}^{u}\right\}\right],\left[\max \left\{v_{1}^{1}, v_{2}^{1}\right\}, \max \left\{v_{1}^{\mathrm{u}}, \mathrm{v}_{2}^{u}\right\}\right]\right)^{\mathrm{c}} \\
& =\left(\left[\max \left\{v_{1}^{1}, v_{2}^{\mathrm{l}}\right\}, \max \left\{v_{1}^{\mathrm{u}}, \mathrm{v}_{2}^{u}\right\}\right],\left[\min \left\{\mu_{1}^{\mathrm{l}}, \mu_{2}^{\mathrm{l}}\right\}, \min \left\{\mu_{1}^{\mathrm{u}}, \mu_{2}^{u}\right\}\right]\right) \\
& \tilde{\mathrm{F}}_{1}{ }^{\mathrm{c}} \cup \tilde{\mathrm{F}}_{2}{ }^{\mathrm{c}}=\left(\left[\mu_{1}^{\mathrm{l}}, \mu_{1}^{\mathrm{u}}\right],\left[\mathrm{v}_{1}^{\mathrm{l}}, \mathrm{v}_{1}^{\mathrm{u}}\right]\right)^{\mathrm{c}} \cup\left(\left[\mu_{2}^{1}, \mu_{2}^{\mathrm{u}}\right],\left[\mathrm{v}_{2}^{\mathrm{l}}, \mathrm{v}_{2}^{\mathrm{u}}\right]\right)^{\mathrm{c}} \\
& =\left(\left[v_{1}^{\mathrm{l}}, \mathrm{v}_{1}^{\mathrm{u}}\right],\left[\mu_{1}^{\mathrm{l}}, \mu_{1}^{\mathrm{u}}\right]\right) \cup\left(\left[v_{2}^{\mathrm{l}}, \mathrm{v}_{2}^{\mathrm{u}}\right],\left[\mu_{2}^{\mathrm{l}}, \mu_{2}^{\mathrm{u}}\right]\right) \\
& =\left(\left[\max \left\{\mathrm{v}_{1}^{\mathrm{l}}, \mathrm{v}_{2}^{\mathrm{l}}\right\}, \max \left\{\mathrm{v}_{1}^{\mathrm{u}}, \mathrm{v}_{2}^{u}\right\}\right],\left[\min \left\{\mu_{1}^{\mathrm{l}}, \mu_{2}^{\mathrm{l}}\right\}, \min \left\{\mu_{1}^{\mathrm{u}}, \mu_{2}^{u}\right\}\right]\right)
\end{aligned}
$$

Thus

$\left(\tilde{\mathrm{F}}_{1} \cap \tilde{\mathrm{F}}_{2}\right)^{\mathrm{c}}=\tilde{\mathrm{F}}_{1}{ }^{\mathrm{c}} \cup \tilde{\mathrm{F}}_{2}{ }^{\mathrm{c}}$

Definition 3.3 Let $\tilde{\mathrm{F}}=\left(\left[\mu^{1}, \mu^{\mathrm{u}}\right],\left[v^{1}, v^{\mathrm{u}}\right]\right), \quad \tilde{\mathrm{F}}_{1}=\left(\left[\mu_{1}^{1}, \mu_{1}^{\mathrm{u}}\right],\left[v_{1}^{1}, v_{1}^{\mathrm{u}}\right]\right)$ and $\tilde{\mathrm{F}}_{2}=$ $\left(\left[\mu_{2}^{1}, \mu_{2}^{u}\right],\left[v_{2}^{1}, v_{2}^{u}\right]\right)$ be three IVFFNs, then the arithmetic operations are defined as follows:

i. $\quad \tilde{\mathrm{F}}_{1} \oplus \tilde{\mathrm{F}}_{2}=\left(\left[\sqrt[3]{\left(\mu_{1}^{\mathrm{l}}\right)^{3}+\left(\mu_{2}^{1}\right)^{3}-\left(\mu_{1}^{\mathrm{l}}\right)^{3}\left(\mu_{2}^{1}\right)^{3}}, \sqrt[3]{\left(\mu_{1}^{\mathrm{u}}\right)^{3}+\left(\mu_{2}^{\mathrm{u}}\right)^{3}-\left(\mu_{1}^{\mathrm{u}}\right)^{3}\left(\mu_{2}^{\mathrm{u}}\right)^{3}}\right],\left[\mathrm{v}_{1}^{\mathrm{l}} \times\right.\right.$

$$
\left.\left.v_{2}^{1}, v_{1}^{u} \times v_{2}^{u}\right]\right)
$$


ii. $\quad \tilde{\mathrm{F}}_{1} \otimes \tilde{\mathrm{F}}_{2}=\left(\left[\mu_{1}^{\mathrm{l}} \times \mu_{2}^{\mathrm{l}}, \mu_{1}^{\mathrm{u}} \times\right.\right.$

$$
\left.\left.\mu_{2}^{\mathrm{u}}\right],\left[\sqrt[3]{\left(v_{1}^{\mathrm{l}}\right)^{3}+\left(v_{2}^{\mathrm{l}}\right)^{3}-\left(v_{1}^{\mathrm{l}}\right)^{3}\left(\mathrm{v}_{2}^{\mathrm{l}}\right)^{3}}, \sqrt[3]{\left(v_{1}^{\mathrm{u}}\right)^{3}+\left(v_{2}^{\mathrm{u}}\right)^{3}-\left(v_{1}^{\mathrm{u}}\right)^{3}\left(\mathrm{v}_{2}^{\mathrm{u}}\right)^{3}}\right]\right)
$$

iii. $\quad \mathrm{k} \tilde{\mathrm{F}}=\left(\left[\sqrt[3]{1-\left(1-\left(\mu^{\mathrm{l}}\right)^{3}\right)^{\mathrm{k}}}, \sqrt[3]{1-\left(1-\left(\mu^{\mathrm{u}}\right)^{3}\right)^{\mathrm{k}}}\right],\left[\left(\mathrm{v}^{\mathrm{l}}\right)^{\mathrm{k}},\left(\mathrm{v}^{\mathrm{u}}\right)^{\mathrm{k}}\right]\right)$, where $\mathrm{k}>0$

iv. $\quad \tilde{\mathrm{F}}^{\mathrm{k}}=\left(\left[\left(\mu^{\mathrm{l}}\right)^{\mathrm{k}},\left(\mu^{\mathrm{u}}\right)^{\mathrm{k}}\right],\left[\sqrt[3]{1-\left(1-\left(v^{\mathrm{l}}\right)^{3}\right)^{\mathrm{k}}}, \sqrt[3]{1-\left(1-\left(v^{\mathrm{u}}\right)^{3}\right)^{\mathrm{k}}}\right]\right)$, where $\mathrm{k}>0$

Theorem 2 Let $\tilde{F}=\left(\left[\mu^{\mathrm{l}}, \mu^{\mathrm{u}}\right],\left[\mathrm{v}^{\mathrm{l}}, v^{\mathrm{u}}\right]\right), \quad \tilde{\mathrm{F}}_{1}=\left(\left[\mu_{1}^{\mathrm{l}}, \mu_{1}^{\mathrm{u}}\right],\left[v_{1}^{\mathrm{l}}, v_{1}^{\mathrm{u}}\right]\right)$ and $\tilde{\mathrm{F}}_{2}=$ $\left(\left[\mu_{2}^{\mathrm{l}}, \mu_{2}^{\mathrm{u}}\right],\left[\mathrm{v}_{2}^{\mathrm{l}}, \mathrm{v}_{2}^{\mathrm{u}}\right]\right)$ be three IVFFNs, then:

i. $\quad \tilde{\mathrm{F}}_{1} \oplus \tilde{\mathrm{F}}_{2}=\tilde{\mathrm{F}}_{2} \oplus \tilde{\mathrm{F}}_{1}$

ii. $\quad \tilde{\mathrm{F}}_{1} \otimes \tilde{\mathrm{F}}_{2}=\tilde{\mathrm{F}}_{2} \otimes \tilde{\mathrm{F}}_{1}$

iii. $\mathrm{k}\left(\tilde{\mathrm{F}}_{1} \oplus \tilde{\mathrm{F}}_{2}\right)=\mathrm{k} \tilde{\mathrm{F}}_{1} \oplus \mathrm{k} \tilde{\mathrm{F}}_{2}$, where $\mathrm{k}>0$

iv. $\left(\mathrm{k}_{1}+\mathrm{k}_{2}\right) \tilde{\mathrm{F}}=\mathrm{k}_{1} \tilde{\mathrm{F}} \oplus \mathrm{k}_{2} \tilde{\mathrm{F}}$, where $\mathrm{k}_{1}>0$ and $\mathrm{k}_{2}>0$

v. $\left(\tilde{\mathrm{F}}_{1} \otimes \tilde{\mathrm{F}}_{2}\right)^{\mathrm{k}}=\tilde{\mathrm{F}}_{2}{ }^{\mathrm{k}} \otimes \tilde{\mathrm{F}}_{1}^{\mathrm{k}}$, where $\mathrm{k}>0$

vi. $\quad \tilde{\mathrm{F}}^{\mathrm{k} 1} \otimes \tilde{\mathrm{F}}^{\mathrm{k} 2}=\tilde{\mathrm{F}}^{\mathrm{k} 1} \quad$, where $\mathrm{k}_{1}>0$ and $\mathrm{k}_{2}>0$

Proof According to definition 3.3 we can obtain:

- $\tilde{\mathrm{F}}_{1} \oplus \tilde{\mathrm{F}}_{2}=\tilde{\mathrm{F}}_{2} \oplus \tilde{\mathrm{F}}_{1}$

$\tilde{\mathrm{F}}_{1} \oplus \tilde{\mathrm{F}}_{2}$

$$
\begin{aligned}
& =\left(\left[\sqrt[3]{\left(\mu_{1}^{\mathrm{l}}\right)^{3}+\left(\mu_{2}^{\mathrm{l}}\right)^{3}-\left(\mu_{1}^{\mathrm{l}}\right)^{3}\left(\mu_{2}^{\mathrm{l}}\right)^{3}}, \sqrt[3]{\left(\mu_{1}^{\mathrm{u}}\right)^{3}+\left(\mu_{2}^{\mathrm{u}}\right)^{3}-\left(\mu_{1}^{\mathrm{u}}\right)^{3}\left(\mu_{2}^{\mathrm{u}}\right)^{3}}\right],\left[v_{1}^{\mathrm{l}} \times v_{2}^{1}, v_{1}^{\mathrm{u}}\right.\right. \\
& \left.\left.\times v_{2}^{u}\right]\right) \\
& =\left(\left[\sqrt[3]{\left(\mu_{2}^{1}\right)^{3}+\left(\mu_{1}^{1}\right)^{3}-\left(\mu_{2}^{1}\right)^{3}\left(\mu_{1}^{1}\right)^{3}}, \sqrt[3]{\left(\mu_{2}^{\mathrm{u}}\right)^{3}+\left(\mu_{1}^{\mathrm{u}}\right)^{3}-\left(\mu_{2}^{\mathrm{u}}\right)^{3}\left(\mu_{1}^{\mathrm{u}}\right)^{3}}\right],\left[v_{2}^{\mathrm{l}} \times v_{1}^{\mathrm{l}}, \mathrm{v}_{2}^{\mathrm{u}}\right.\right. \\
& \left.\left.\times v_{1}^{\mathrm{u}}\right]\right) \\
& =\tilde{\mathrm{F}}_{2} \oplus \tilde{\mathrm{F}}_{1}
\end{aligned}
$$


- $\tilde{\mathrm{F}}_{1} \otimes \tilde{\mathrm{F}}_{2}=\tilde{\mathrm{F}}_{2} \otimes \tilde{\mathrm{F}}_{1}$

$\tilde{\mathrm{F}}_{1} \otimes \tilde{\mathrm{F}}_{2}$

$$
\begin{aligned}
& =\left(\left[\mu_{1}^{\mathrm{l}} \times \mu_{2}^{\mathrm{l}}, \mu_{1}^{\mathrm{u}}\right.\right. \\
& \left.\left.\times \mu_{2}^{\mathrm{u}}\right],\left[\sqrt[3]{\left(\mathrm{v}_{1}^{\mathrm{l}}\right)^{3}+\left(v_{2}^{\mathrm{l}}\right)^{3}-\left(v_{1}^{\mathrm{l}}\right)^{3}\left(v_{2}^{\mathrm{l}}\right)^{3}}, \sqrt[3]{\left(v_{1}^{\mathrm{u}}\right)^{3}+\left(v_{2}^{\mathrm{u}}\right)^{3}-\left(v_{1}^{\mathrm{u}}\right)^{3}\left(v_{2}^{\mathrm{u}}\right)^{3}}\right]\right) \\
& =\left(\left[\mu_{2}^{\mathrm{l}} \times \mu_{1}^{\mathrm{l}}, \mu_{2}^{\mathrm{u}}\right.\right. \\
& \left.\left.\times \mu_{1}^{\mathrm{u}}\right],\left[\sqrt[3]{\left(v_{2}^{\mathrm{l}}\right)^{3}+\left(v_{1}^{\mathrm{l}}\right)^{3}-\left(v_{2}^{\mathrm{l}}\right)^{3}\left(v_{1}^{\mathrm{l}}\right)^{3}}, \sqrt[3]{\left(v_{2}^{\mathrm{u}}\right)^{3}+\left(v_{1}^{\mathrm{u}}\right)^{3}-\left(v_{2}^{\mathrm{u}}\right)^{3}\left(v_{1}^{\mathrm{u}}\right)^{3}}\right]\right) \\
& =\tilde{\mathrm{F}}_{2} \otimes \tilde{\mathrm{F}}_{1}
\end{aligned}
$$

- $\mathrm{k}\left(\tilde{\mathrm{F}}_{1} \oplus \tilde{\mathrm{F}}_{2}\right)=\mathrm{k} \tilde{\mathrm{F}}_{1} \oplus \mathrm{k} \tilde{\mathrm{F}}_{2}$, where $\mathrm{k}>0$

$$
\begin{aligned}
& \mathrm{k}\left(\tilde{\mathrm{F}}_{1} \oplus \tilde{\mathrm{F}}_{2}\right)=\mathrm{k}\left(\left[\sqrt[3]{\left(\mu_{1}^{\mathrm{l}}\right)^{3}+\left(\mu_{2}^{\mathrm{l}}\right)^{3}-\left(\mu_{1}^{\mathrm{l}}\right)^{3}\left(\mu_{2}^{\mathrm{l}}\right)^{3}}, \sqrt[3]{\left(\mu_{1}^{\mathrm{u}}\right)^{3}+\left(\mu_{2}^{\mathrm{u}}\right)^{3}-\left(\mu_{1}^{\mathrm{u}}\right)^{3}\left(\mu_{2}^{\mathrm{u}}\right)^{3}}\right],\left[\mathrm{U}_{1}^{\mathrm{l}} \times\right.\right. \\
& \left.\left.u_{2}^{1}, \cup_{1}^{\mathrm{u}} \times u_{2}^{\mathrm{u}}\right]\right)
\end{aligned}
$$

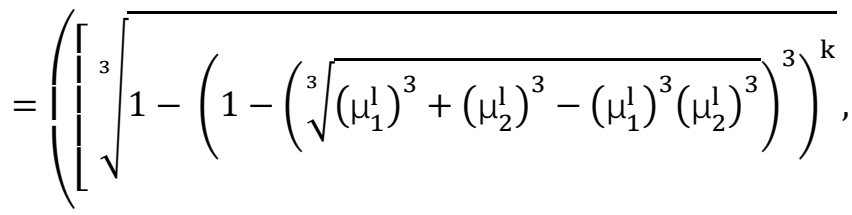

$$
\begin{aligned}
& \left.\left.\sqrt[3]{1-\left(1-\left(\sqrt[3]{\left(\mu_{1}^{\mathrm{u}}\right)^{3}+\left(\mu_{2}^{\mathrm{u}}\right)^{3}-\left(\mu_{1}^{\mathrm{u}}\right)^{3}\left(\mu_{2}^{\mathrm{u}}\right)^{3}}\right)^{3}\right)^{\mathrm{k}}}\right],\left[\left(v_{1}^{\mathrm{l}} \times v_{2}^{\mathrm{l}}\right)^{\mathrm{k}},\left(\mathrm{v}_{1}^{\mathrm{u}} \times v_{2}^{\mathrm{u}}\right)^{\mathrm{k}}\right]\right) \\
& =\left(\left[\sqrt[3]{1-\left(1-\left(\mu_{1}^{1}\right)^{3}+\left(\mu_{2}^{l}\right)^{3}-\left(\mu_{1}^{l}\right)^{3}\left(\mu_{2}^{1}\right)^{3}\right)^{\mathrm{k}}},\right.\right. \\
& \left.\left.\sqrt[3]{1-\left(1-\left(\mu_{1}^{\mathrm{u}}\right)^{3}+\left(\mu_{2}^{\mathrm{u}}\right)^{3}-\left(\mu_{1}^{\mathrm{u}}\right)^{3}\left(\mu_{2}^{\mathrm{u}}\right)^{3}\right)^{\mathrm{k}}}\right],\left[\left(\mathrm{u}_{1}^{\mathrm{l}} \times \mathrm{u}_{2}^{\mathrm{l}}\right)^{\mathrm{k}},\left(\mathrm{u}_{1}^{\mathrm{u}} \times \mathrm{u}_{2}^{\mathrm{u}}\right)^{\mathrm{k}}\right]\right) \\
& \mathrm{k} \tilde{\mathrm{F}}_{1} \oplus \mathrm{k} \tilde{\mathrm{F}}_{2}
\end{aligned}
$$




$$
\begin{aligned}
& =\left(\left[\sqrt[3]{1-\left(1-\left(\mu_{1}^{\mathrm{l}}\right)^{3}\right)^{\mathrm{k}}}, \sqrt[3]{1-\left(1-\left(\mu_{1}^{\mathrm{u}}\right)^{3}\right)^{\mathrm{k}}}\right],\left[\left(\mathrm{u}_{1}^{\mathrm{l}}\right)^{\mathrm{k}},\left(\mathrm{u}_{1}^{\mathrm{u}}\right)^{\mathrm{k}}\right]\right) \oplus \\
& \left(\left[\sqrt[3]{1-\left(1-\left(\mu_{2}^{\mathrm{l}}\right)^{3}\right)^{\mathrm{k}}}, \sqrt[3]{1-\left(1-\left(\mu_{2}^{\mathrm{u}}\right)^{3}\right)^{\mathrm{k}}}\right],\left[\left(\mathrm{u}_{2}^{\mathrm{l}}\right)^{\mathrm{k}},\left(\mathrm{u}_{2}^{\mathrm{u}}\right)^{\mathrm{k}}\right]\right) \\
& = \\
& \left(\left[\sqrt[3]{\left(1-\left(1-\left(\mu_{1}^{\mathrm{l}}\right)^{3}\right)^{\mathrm{k}}\right)+\left(1-\left(1-\left(\mu_{2}^{\mathrm{l}}\right)^{3}\right)^{\mathrm{k}}\right)-\left(1-\left(1-\left(\mu_{1}^{\mathrm{l}}\right)^{3}\right)^{\mathrm{k}}\right)\left(1-\left(1-\left(\mu_{2}^{\mathrm{l}}\right)^{3}\right)^{\mathrm{k}}\right)},\right.\right. \\
& \left.\sqrt[3]{\left(1-\left(1-\left(\mu_{1}^{\mathrm{u}}\right)^{3}\right)^{\mathrm{k}}\right)+\left(1-\left(1-\left(\mu_{2}^{\mathrm{u}}\right)^{3}\right)^{\mathrm{k}}\right)-\left(1-\left(1-\left(\mu_{1}^{\mathrm{u}}\right)^{3}\right)^{\mathrm{k}}\right)\left(1-\left(1-\left(\mu_{2}^{\mathrm{u}}\right)^{3}\right)^{\mathrm{k}}\right)}\right], \\
& \left.\left[\left(u_{1}^{\mathrm{l}}\right)^{\mathrm{k}} \times\left(\mathrm{u}_{2}^{\mathrm{l}}\right)^{\mathrm{k}},\left(\mathrm{u}_{1}^{\mathrm{u}}\right)^{\mathrm{k}} \times\left(\mathrm{u}_{2}^{\mathrm{u}}\right)^{\mathrm{k}}\right]\right) \\
& =\left(\left[\sqrt[3]{1-\left(1-\left(\mu_{1}^{1}\right)^{3}+\left(\mu_{2}^{1}\right)^{3}-\left(\mu_{1}^{1}\right)^{3}\left(\mu_{2}^{1}\right)^{3}\right)^{\mathrm{k}}},\right.\right. \\
& \left.\left.\sqrt[3]{1-\left(1-\left(\mu_{1}^{\mathrm{u}}\right)^{3}+\left(\mu_{2}^{\mathrm{u}}\right)^{3}-\left(\mu_{1}^{\mathrm{u}}\right)^{3}\left(\mu_{2}^{\mathrm{u}}\right)^{3}\right)^{\mathrm{k}}}\right],\left[\left(\mathrm{u}_{1}^{\mathrm{l}} \times \mathrm{u}_{2}^{\mathrm{l}}\right)^{\mathrm{k}},\left(\mathrm{u}_{1}^{\mathrm{u}} \times \mathrm{u}_{2}^{\mathrm{u}}\right)^{\mathrm{k}}\right]\right)
\end{aligned}
$$

Thus:

$\mathrm{k}\left(\widetilde{\mathrm{F}}_{1} \oplus \tilde{\mathrm{F}}_{2}\right)=\mathrm{k} \widetilde{\mathrm{F}}_{1} \oplus \mathrm{k} \widetilde{\mathrm{F}}_{2}$

- $\left(\mathrm{k}_{1}+\mathrm{k}_{2}\right) \tilde{\mathrm{F}}=\mathrm{k}_{1} \tilde{\mathrm{F}} \oplus \mathrm{k}_{2} \tilde{\mathrm{F}}$, where $\mathrm{k}_{1}>0$ and $\mathrm{k}_{2}>0$

$\left(\mathrm{k}_{1}+\mathrm{k}_{2}\right) \tilde{\mathrm{F}}=\left(\mathrm{k}_{1}+\mathrm{k}_{2}\right)\left(\left[\mu^{\mathrm{l}}, \mu^{\mathrm{u}}\right],\left[\mathrm{v}^{\mathrm{l}}, \mathrm{v}^{\mathrm{u}}\right]\right)$

$=\left(\left[\sqrt[3]{1-\left(1-\left(\mu^{\mathrm{l}}\right)^{3}\right)^{\left(\mathrm{k}_{1}+\mathrm{k}_{2}\right)}}, \sqrt[3]{1-\left(1-\left(\mu^{\mathrm{u}}\right)^{3}\right)^{\left(\mathrm{k}_{1}+\mathrm{k}_{2}\right)}}\right],\left[\left(\mathrm{v}^{\mathrm{l}}\right)^{\left(\mathrm{k}_{1}+\mathrm{k}_{2}\right)},\left(v^{\mathrm{u}}\right)^{\left(\mathrm{k}_{1}+\mathrm{k}_{2}\right)}\right]\right)$

$=\left(\left[\sqrt[3]{1-\left(1-\left(\mu^{\mathrm{l}}\right)^{3}\right)^{\mathrm{k} 1}\left(1-\left(\mu^{\mathrm{l}}\right)^{3}\right)^{\mathrm{k} 2}}, \sqrt[3]{1-\left(1-\left(\mu^{\mathrm{u}}\right)^{3}\right)^{\mathrm{k} 1}\left(1-\left(\mu^{\mathrm{u}}\right)^{3}\right)^{\mathrm{k} 2}}\right]\right.$,

$\left.\left[\left(u^{\mathrm{l}}\right)^{\mathrm{k} 1}\left(v^{\mathrm{l}}\right)^{\mathrm{k} 2},\left(v^{\mathrm{u}}\right)^{\mathrm{k} 1}\left(v^{\mathrm{u}}\right)^{\mathrm{k} 2}\right]\right)$

$\mathrm{k}_{1} \tilde{\mathrm{F}} \oplus \mathrm{k}_{2} \tilde{\mathrm{F}}=\left(\left[\sqrt[3]{1-\left(1-\left(\mu^{\mathrm{l}}\right)^{3}\right)^{\mathrm{k} 1}}, \sqrt[3]{1-\left(1-\left(\mu^{\mathrm{u}}\right)^{3}\right)^{\mathrm{k} 1}}\right],\left[\left(v^{\mathrm{l}}\right)^{\mathrm{k} 1},\left(\mathrm{v}^{\mathrm{u}}\right)^{\mathrm{k} 1}\right]\right)$

$$
\oplus\left(\left[\sqrt[3]{1-\left(1-\left(\mu^{\mathrm{l}}\right)^{3}\right)^{\mathrm{k} 2}}, \sqrt[3]{1-\left(1-\left(\mu^{\mathrm{u}}\right)^{3}\right)^{\mathrm{k} 2}}\right],\left[\left(v^{\mathrm{l}}\right)^{\mathrm{k} 2},\left(v^{\mathrm{u}}\right)^{\mathrm{k} 2}\right]\right)
$$


$\left(\left[\sqrt[3]{1-\left(1-\left(\mu^{\mathrm{l}}\right)^{3}\right)^{\mathrm{k} 1}+1-\left(1-\left(\mu^{\mathrm{l}}\right)^{3}\right)^{\mathrm{k} 2}-\left(\left(1-\left(1-\left(\mu^{\mathrm{l}}\right)^{3}\right)^{\mathrm{k} 1}\right)\left(1-\left(1-\left(\mu^{\mathrm{l}}\right)^{3}\right)^{\mathrm{k} 2}\right)\right)}\right.\right.$,

$\sqrt[3]{1-\left(1-\left(\mu^{\mathrm{u}}\right)^{3}\right)^{\mathrm{k} 1}+1-\left(1-\left(\mu^{\mathrm{u}}\right)^{3}\right)^{\mathrm{k} 2}-\left(\left(1-\left(1-\left(\mu^{\mathrm{u}}\right)^{3}\right)^{\mathrm{k} 1}\right)\left(1-\left(1-\left(\mu^{\mathrm{u}}\right)^{3}\right)^{\mathrm{k} 2}\right)\right)}$,

$\left.\left[\left(u^{\mathrm{l}}\right)^{\mathrm{k} 1} \times\left(\mathrm{u}^{\mathrm{l}}\right)^{\mathrm{k} 2},\left(\mathrm{u}^{\mathrm{u}}\right)^{\mathrm{k} 1} \times\left(\mathrm{u}^{\mathrm{u}}\right)^{\mathrm{k} 2}\right]\right)$

$=\left(\left[\sqrt[3]{1-\left(1-\left(\mu^{\mathrm{l}}\right)^{3}\right)^{\mathrm{k} 1}\left(1-\left(\mu^{\mathrm{l}}\right)^{3}\right)^{\mathrm{k} 2}}, \sqrt[3]{1-\left(1-\left(\mu^{\mathrm{u}}\right)^{3}\right)^{\mathrm{k} 1}\left(1-\left(\mu^{\mathrm{u}}\right)^{3}\right)^{\mathrm{k} 2}}\right]\right.$,

$\left.\left[\left(v^{1}\right)^{\mathrm{k} 1}\left(\mathrm{v}^{\mathrm{l}}\right)^{\mathrm{k} 2},\left(\mathrm{v}^{\mathrm{u}}\right)^{\mathrm{k} 1}\left(\mathrm{v}^{\mathrm{u}}\right)^{\mathrm{k} 2}\right]\right)$

Thus

$\left(\mathrm{k}_{1}+\mathrm{k}_{2}\right) \tilde{\mathrm{F}}=\mathrm{k}_{1} \tilde{\mathrm{F}} \oplus \mathrm{k}_{2} \tilde{\mathrm{F}}$

- $\left(\tilde{\mathrm{F}}_{1} \otimes \tilde{\mathrm{F}}_{2}\right)^{\mathrm{k}}=\tilde{\mathrm{F}}_{1}^{\mathrm{k}} \otimes \tilde{\mathrm{F}}_{2}{ }^{\mathrm{k}}$, where $\mathrm{k}>0$

$\left(\tilde{\mathrm{F}}_{1} \otimes \tilde{\mathrm{F}}_{2}\right)^{\mathrm{k}}=\left(\left[\mu_{1}^{\mathrm{l}} \times \mu_{2}^{\mathrm{l}}, \mu_{1}^{\mathrm{u}} \times \mu_{2}^{\mathrm{u}}\right],\left[\sqrt[3]{\left(v_{1}^{\mathrm{l}}\right)^{3}+\left(\mathrm{v}_{2}^{\mathrm{l}}\right)^{3}-\left(v_{1}^{\mathrm{l}}\right)^{3}\left(v_{2}^{\mathrm{l}}\right)^{3}}\right.\right.$,

$$
\left.\left.\sqrt[3]{\left(v_{1}^{u}\right)^{3}+\left(v_{2}^{u}\right)^{3}-\left(v_{1}^{u}\right)^{3}\left(v_{2}^{u}\right)^{3}}\right]\right)^{k}
$$

$=\left(\left[\left(\mu_{1}^{\mathrm{l}} \times \mu_{2}^{\mathrm{l}}\right)^{\mathrm{k}},\left(\mu_{1}^{\mathrm{u}} \times \mu_{2}^{\mathrm{u}}\right)^{\mathrm{k}}\right],\left[\sqrt[3]{1-\left(1-\left(v_{1}^{\mathrm{l}}\right)^{3}+\left(v_{2}^{\mathrm{l}}\right)^{3}-\left(v_{1}^{\mathrm{l}}\right)^{3}\left(v_{2}^{\mathrm{l}}\right)^{3}\right)^{\mathrm{k}}}\right.\right.$,

$\left.\left.\sqrt[3]{1-\left(1-\left(v_{1}^{\mathrm{u}}\right)^{3}+\left(\mathrm{v}_{2}^{\mathrm{u}}\right)^{3}-\left(\mathrm{v}_{1}^{\mathrm{u}}\right)^{3}\left(\mathrm{v}_{2}^{\mathrm{u}}\right)^{3}\right)^{\mathrm{k}}}\right]\right)$

$=\left(\left[\left(\mu_{1}^{\mathrm{l}}\right)^{\mathrm{k}} \times\left(\mu_{2}^{\mathrm{l}}\right)^{\mathrm{k}},\left(\mu_{1}^{\mathrm{u}}\right)^{\mathrm{k}} \times\left(\mu_{2}^{\mathrm{u}}\right)^{\mathrm{k}}\right],\left[\sqrt[3]{1-\left(1-\left(v_{1}^{\mathrm{l}}\right)^{3}\right)^{\mathrm{k}}\left(1-\left(v_{2}^{\mathrm{l}}\right)^{3}\right)^{\mathrm{k}}}\right.\right.$,

$\left.\left.\sqrt[3]{1-\left(1-\left(v_{1}^{\mathrm{u}}\right)^{3}\right)^{\mathrm{k}}\left(1-\left(\mathrm{v}_{2}^{\mathrm{u}}\right)^{3}\right)^{\mathrm{k}}}\right]\right)$

$=\tilde{\mathrm{F}}_{1}^{\mathrm{k}} \otimes \tilde{\mathrm{F}}_{2}^{\mathrm{k}}$ 
- $\tilde{\mathrm{F}}^{\mathrm{k} 1} \otimes \tilde{\mathrm{F}}^{\mathrm{k} 2}=\tilde{\mathrm{F}}^{\mathrm{k} 1+\mathrm{k} 2}$, where $\mathrm{k}_{1}>0$ and $\mathrm{k}_{2}>0$

$\tilde{\mathrm{F}}^{\mathrm{k} 1} \otimes \tilde{\mathrm{F}}^{\mathrm{k} 2}$

$$
\begin{aligned}
& =\left(\left[\left(\mu^{\mathrm{l}}\right)^{\mathrm{k} 1},\left(\mu^{\mathrm{u}}\right)^{\mathrm{k} 1}\right],\left[\sqrt[3]{1-\left(1-\left(v^{\mathrm{l}}\right)^{3}\right)^{\mathrm{k} 1}}, \sqrt[3]{1-\left(1-\left(v^{\mathrm{u}}\right)^{3}\right)^{\mathrm{k} 1}}\right]\right) \\
& \otimes\left(\left[\left(\mu^{\mathrm{l}}\right)^{\mathrm{k} 2},\left(\mu^{\mathrm{u}}\right)^{\mathrm{k} 2}\right],\left[\sqrt[3]{1-\left(1-\left(v^{\mathrm{l}}\right)^{3}\right)^{\mathrm{k} 2}}, \sqrt[3]{1-\left(1-\left(v^{\mathrm{u}}\right)^{3}\right)^{\mathrm{k} 2}}\right]\right)
\end{aligned}
$$$$
=\left(\left[\left(\mu^{\mathrm{l}}\right)^{\mathrm{k} 1} \times\left(\mu^{\mathrm{l}}\right)^{\mathrm{k} 2},\left(\mu^{\mathrm{u}}\right)^{\mathrm{k} 1} \times\left(\mu^{\mathrm{u}}\right)^{\mathrm{k} 2}\right],\right.
$$$$
\left[\sqrt[3]{1-\left(1-\left(v^{\mathrm{l}}\right)^{3}\right)^{\mathrm{k} 1}+1-\left(1-\left(\mathrm{v}^{\mathrm{l}}\right)^{3}\right)^{\mathrm{k} 2}-\left(\left(1-\left(1-\left(\mathrm{v}^{\mathrm{l}}\right)^{3}\right)^{\mathrm{k} 1}\right)\left(1-\left(1-\left(\mathrm{v}^{\mathrm{l}}\right)^{3}\right)^{\mathrm{k} 2}\right)\right)},\right.
$$

$\left.\left.\sqrt[3]{1-\left(1-\left(v^{\mathrm{u}}\right)^{3}\right)^{\mathrm{k} 1}+1-\left(1-\left(\mathrm{v}^{\mathrm{u}}\right)^{3}\right)^{\mathrm{k} 2}-\left(\left(1-\left(1-\left(\mathrm{u}^{\mathrm{u}}\right)^{3}\right)^{\mathrm{k} 1}\right)\left(1-\left(1-\left(\mathrm{v}^{\mathrm{u}}\right)^{3}\right)^{\mathrm{k} 2}\right)\right)}\right]\right)$

$=\left(\left[\left(\mu^{\mathrm{l}}\right)^{\mathrm{k} 1+\mathrm{k} 2},\left(\mu^{\mathrm{u}}\right)^{\mathrm{k} 1+\mathrm{k}}\right],\left[\sqrt[3]{1-\left(1-\left(\mathrm{v}^{\mathrm{l}}\right)^{3}\right)^{\mathrm{k} 1+\mathrm{k} 2}}, \sqrt[3]{1-\left(1-\left(v^{\mathrm{u}}\right)^{3}\right)^{\mathrm{k} 1+\mathrm{k} 2}}\right]\right)$

$=\tilde{\mathrm{F}}^{\mathrm{k} 1+\mathrm{k} 2}$

In many decision-making problems, the distance between two fuzzy sets is calculated using Hamming distance due to its simple calculation. So, we define the Hamming distance between two interval-valued fermatean fuzzy numbers and call it the interval-valued fermatean fuzzy Hamming distance (IVFFHD).

Definition 3.4 Let $\widetilde{F}_{1}=\left(\left[\mu_{1}^{\mathrm{l}}, \mu_{1}^{\mathrm{u}}\right],\left[v_{1}^{\mathrm{l}}, \mathrm{v}_{1}^{\mathrm{u}}\right]\right)$ and $\widetilde{\mathrm{F}}_{2}=\left(\left[\mu_{2}^{\mathrm{l}}, \mu_{2}^{\mathrm{u}}\right],\left[\mathrm{v}_{2}^{\mathrm{l}}, \mathrm{v}_{2}^{\mathrm{u}}\right]\right)$ be two IVFFNs, then we can obtain the IVFFHD:

$d_{\text {IVFFHD }}\left(\tilde{\mathrm{F}}_{1}, \tilde{\mathrm{F}}_{2}\right)$

$$
\begin{aligned}
& =\frac{1}{4}\left(\left|\left(\mu_{1}^{\mathrm{l}}\right)^{3}-\left(\mu_{2}^{\mathrm{l}}\right)^{3}\right|+\left|\left(\mu_{1}^{\mathrm{u}}\right)^{3}-\left(\mu_{2}^{\mathrm{u}}\right)^{3}\right|+\left|\left(v_{1}^{\mathrm{l}}\right)^{3}-\left(v_{2}^{\mathrm{l}}\right)^{3}\right|\right. \\
& \left.+\left|\left(v_{1}^{\mathrm{u}}\right)^{3}-\left(v_{2}^{\mathrm{u}}\right)^{3}\right|+\left|\left(\pi_{1}^{\mathrm{l}}\right)^{3}-\left(\pi_{2}^{\mathrm{l}}\right)^{3}\right|+\left|\left(\pi_{1}^{\mathrm{u}}\right)^{3}-\left(\pi_{2}^{\mathrm{u}}\right)^{3}\right|\right)
\end{aligned}
$$

Where, $\pi_{i}^{\mathrm{l}}=\left(1-\left(\mu_{i}^{\mathrm{l}}\right)^{3}-\left(v_{i}^{\mathrm{l}}\right)^{3}\right)^{\frac{1}{3}}, \mathrm{i}=1,2$ and $\pi_{i}^{\mathrm{u}}=\left(1-\left(\mu_{i}^{\mathrm{u}}\right)^{3}-\left(v_{i}^{\mathrm{u}}\right)^{3}\right)^{\frac{1}{3}}, \mathrm{i}=1,2$ 
Definition 3.5 Let $\tilde{F}=\left(\left[\mu^{1}, \mu^{\mathrm{u}}\right],\left[v^{\mathrm{l}}, v^{\mathrm{u}}\right]\right)$ be a IVFFN, then the score function of $\tilde{\mathrm{F}}$ can be defined as

$\operatorname{score}(\widetilde{\mathrm{F}})=\frac{\left(\mu^{\mathrm{l}}\right)^{3}+\left(\mu^{\mathrm{u}}\right)^{3}-\left(v^{\mathrm{l}}\right)^{3}-\left(v^{\mathrm{u}}\right)^{3}}{2}$

Based on the arithmetic operations of IVFFN's, we introduce an aggregation operator, which is the Interval-valued Fermatean Fuzzy Weighted Averaging (IVFFWA) operator.

Definition 3.5 Let $\tilde{\mathrm{F}}_{\mathrm{k}}=\left(\left[\mu_{\mathrm{k}}^{\mathrm{l}}, \mu_{\mathrm{k}}^{\mathrm{u}}\right],\left[v_{\mathrm{k}}^{\mathrm{l}}, \mathrm{v}_{\mathrm{k}}^{\mathrm{u}}\right]\right), \mathrm{k}=1,2, \ldots \mathrm{N}$ be a collection of IVFFN's then the $\operatorname{IVFFWA}\left(\tilde{\mathrm{F}}_{1}, \tilde{\mathrm{F}}_{2}, \ldots \ldots, \tilde{\mathrm{F}}_{\mathrm{N}}\right)=w_{1} \otimes \tilde{\mathrm{F}}_{1} \oplus w_{2} \otimes \tilde{\mathrm{F}}_{2} \oplus \ldots \ldots \oplus w_{\mathrm{N}} \otimes \tilde{\mathrm{F}}_{\mathrm{N}}$ Where $w_{1}, w_{2} \ldots \ldots w_{\mathrm{N}}$ are the weights of $\tilde{\mathrm{F}}_{1}, \tilde{\mathrm{F}}_{2}, \ldots \ldots, \widetilde{\mathrm{F}}_{\mathrm{N}}$ such that $0 \leq \mathrm{w}_{\mathrm{k}} \leq 1$ and $\sum_{k=1}^{\mathrm{N}} \mathrm{w}_{\mathrm{k}}$

Theorem 3 Let $\tilde{\mathrm{F}}_{\mathrm{k}}=\left(\left[\mu_{\mathrm{k}}^{\mathrm{l}}, \mu_{\mathrm{k}}^{\mathrm{u}}\right],\left[\mathrm{v}_{\mathrm{k}}^{\mathrm{l}}, \mathrm{v}_{\mathrm{k}}^{\mathrm{u}}\right]\right), \mathrm{k}=1,2, \ldots \mathrm{N}$ be a collection of IVFFN's then from definition 3.5.

$\operatorname{IVFFWA}\left(\tilde{\mathrm{F}}_{1}, \tilde{\mathrm{F}}_{2}, \ldots \ldots, \tilde{\mathrm{F}}_{\mathrm{N}}\right)=$

$\left(\left[\sqrt[3]{1-\prod_{\mathrm{k}=1}^{\mathrm{N}}\left(1-\left(\mu_{\mathrm{k}}^{\mathrm{l}}\right)^{3}\right)^{\mathrm{w}_{\mathrm{k}}}}, \sqrt[3]{\left(1-\prod_{\mathrm{k}=1}^{\mathrm{N}}\left(1-\left(\mu_{\mathrm{k}}^{\mathrm{u}}\right)^{3}\right)^{\mathrm{w}_{\mathrm{k}}}\right)}\right]\right.$

$\left.\left[\left(\prod_{\mathrm{k}=1}^{\mathrm{N}}\left(\mathrm{v}_{\mathrm{k}}^{\mathrm{l}}\right)^{\mathrm{w}_{\mathrm{k}}}\right),\left(\prod_{\mathrm{k}=1}^{\mathrm{N}}\left(\mathrm{v}_{\mathrm{k}}^{\mathrm{u}}\right)^{\mathrm{w}_{\mathrm{k}}}\right)\right]\right)$

Proof We can prove the above theorem using mathematical induction method.

For two IVFFN's

$w_{1} \otimes \tilde{\mathrm{F}}_{1}=\left(\left[\sqrt[3]{1-\left(1-\left(\mu_{1}^{\mathrm{l}}\right)^{3}\right)^{w_{1}}}, \sqrt[3]{1-\left(1-\left(\mu_{1}^{\mathrm{u}}\right)^{3}\right)^{w_{1}}}\right],\left[\left(v_{1}^{\mathrm{l}}\right)^{w_{1}},\left(\mathrm{v}_{1}^{\mathrm{u}}\right)^{w_{1}}\right]\right)$ and
$w_{2} \otimes \tilde{\mathrm{F}}_{2}=\left(\left[\sqrt[3]{1-\left(1-\left(\mu_{2}^{\mathrm{l}}\right)^{3}\right)^{w_{2}}}, \sqrt[3]{1-\left(1-\left(\mu_{2}^{\mathrm{u}}\right)^{3}\right)^{w_{2}}}\right],\left[\left(v_{2}^{\mathrm{l}}\right)^{w_{2}},\left(v_{2}^{\mathrm{u}}\right)^{w_{2}}\right]\right)$

$\operatorname{IVFFWA}\left(\tilde{\mathrm{F}}_{1}, \tilde{\mathrm{F}}_{2}\right)=w_{1} \otimes \tilde{\mathrm{F}}_{1} \oplus w_{2} \otimes \tilde{\mathrm{F}}_{2}$ 


$$
\begin{aligned}
& =\left(\left[\sqrt[3]{1-\left(1-\left(\mu_{1}^{\mathrm{l}}\right)^{3}\right)^{w_{1}}}, \sqrt[3]{1-\left(1-\left(\mu_{1}^{\mathrm{u}}\right)^{3}\right)^{w_{1}}}\right],\left[\left(v_{1}^{\mathrm{l}}\right)^{w_{1}},\left(v_{1}^{\mathrm{u}}\right)^{w_{1}}\right]\right) \oplus \\
& \left(\left[\sqrt[3]{1-\left(1-\left(\mu_{2}^{1}\right)^{3}\right)^{w_{2}}}, \sqrt[3]{1-\left(1-\left(\mu_{2}^{\mathrm{u}}\right)^{3}\right)^{w_{2}}}\right],\left[\left(v_{2}^{1}\right)^{w_{2}},\left(v_{2}^{\mathrm{u}}\right)^{w_{2}}\right]\right) \\
& = \\
& \left(\left[\sqrt[3]{\left(1-\left(1-\left(\mu_{1}^{1}\right)^{3}\right)^{w_{1}}\right)+\left(1-\left(1-\left(\mu_{2}^{1}\right)^{3}\right)^{w_{2}}\right)-\left(1-\left(1-\left(\mu_{1}^{1}\right)^{3}\right)^{w_{1}}\right)\left(1-\left(1-\left(\mu_{2}^{1}\right)^{3}\right)^{w_{2}}\right)},\right.\right. \\
& \left.\sqrt[3]{\left(1-\left(1-\left(\mu_{1}^{\mathrm{u}}\right)^{3}\right)^{w_{1}}\right)+\left(1-\left(1-\left(\mu_{2}^{u}\right)^{3}\right)^{w_{2}}\right)-\left(1-\left(1-\left(\mu_{1}^{\mathrm{u}}\right)^{3}\right)^{w_{1}}\right)\left(1-\left(1-\left(\mu_{2}^{\mathrm{u}}\right)^{3}\right)^{w_{2}}\right)}\right],\left[\left(v_{1}^{\mathrm{l}}\right)^{w_{1}} \times\right. \\
& \left.\left.\left.\left(\mathrm{v}_{2}^{\mathrm{l}}\right)^{w_{2}},\left(\mathrm{v}_{1}^{\mathrm{u}}\right)^{w_{1}} \times\left(\mathrm{u}_{2}^{\mathrm{u}}\right)^{w_{2}}\right]\right]\right) \\
& = \\
& \left(\left[\sqrt[3]{1-\left(1-\left(\mu_{1}^{1}\right)^{3}\right)^{w_{1}}\left(1-\left(\mu_{2}^{1}\right)^{3}\right)^{w_{2}}}, \sqrt[3]{1-\left(1-\left(\mu_{1}^{\mathrm{u}}\right)^{3}\right)^{w_{1}}\left(1-\left(\mu_{2}^{u}\right)^{3}\right)^{w_{2}}}\right],\left[\left(v_{1}^{1}\right)^{w_{1}} \times\right.\right. \\
& \left.\left.\left.\left(\mathrm{v}_{2}^{\mathrm{l}}\right)^{w_{2}},\left(\mathrm{v}_{1}^{\mathrm{u}}\right)^{w_{1}} \times\left(\mathrm{v}_{2}^{\mathrm{u}}\right)^{w_{2}}\right]\right]\right) \\
& = \\
& \left(\left[\sqrt[3]{1-\prod_{\mathrm{k}=1}^{2}\left(1-\left(\mu_{\mathrm{k}}^{\mathrm{l}}\right)^{3}\right)^{\mathrm{w}_{\mathrm{k}}}}, \sqrt[3]{\left(1-\prod_{\mathrm{k}=1}^{2}\left(1-\left(\mu_{\mathrm{k}}^{\mathrm{u}}\right)^{3}\right)^{\mathrm{w}_{\mathrm{k}}}\right)}\right],\left[\left(\prod_{\mathrm{k}=1}^{3}\left(v_{\mathrm{k}}^{\mathrm{l}}\right)^{\mathrm{w}_{\mathrm{k}}}\right),\left(\prod_{\mathrm{k}=1}^{3}\left(v_{\mathrm{k}}^{\mathrm{u}}\right)^{\mathrm{w}_{\mathrm{k}}}\right)\right]\right)
\end{aligned}
$$

For three IVFFN's:

$$
\begin{aligned}
& w_{1} \otimes \tilde{\mathrm{F}}_{1}=\left(\left[\sqrt[3]{1-\left(1-\left(\mu_{1}^{1}\right)^{3}\right)^{w_{1}}}, \sqrt[3]{1-\left(1-\left(\mu_{1}^{\mathrm{u}}\right)^{3}\right)^{w_{1}}}\right],\left[\left(v_{1}^{\mathrm{l}}\right)^{w_{1}},\left(\mathrm{v}_{1}^{\mathrm{u}}\right)^{w_{1}}\right]\right), \\
& w_{2} \otimes \tilde{\mathrm{F}}_{2}=\left(\left[\sqrt[3]{1-\left(1-\left(\mu_{2}^{\mathrm{l}}\right)^{3}\right)^{w_{2}}}, \sqrt[3]{1-\left(1-\left(\mu_{2}^{\mathrm{u}}\right)^{3}\right)^{w_{2}}}\right],\left[\left(v_{2}^{\mathrm{l}}\right)^{w_{2}},\left(\mathrm{v}_{2}^{\mathrm{u}}\right)^{w_{2}}\right]\right) \text { and } \\
& w_{3} \otimes \tilde{\mathrm{F}}_{3}=\left(\left[\sqrt[3]{1-\left(1-\left(\mu_{3}^{\mathrm{l}}\right)^{3}\right)^{w_{3}}}, \sqrt[3]{1-\left(1-\left(\mu_{3}^{\mathrm{u}}\right)^{3}\right)^{w_{3}}}\right],\left[\left(v_{3}^{\mathrm{l}}\right)^{w_{3}},\left(\mathrm{v}_{3}^{\mathrm{u}}\right)^{w_{3}}\right]\right)
\end{aligned}
$$

$\operatorname{IVFFWA}\left(\tilde{\mathrm{F}}_{1}, \tilde{\mathrm{F}}_{2}, \tilde{\mathrm{F}}_{3}\right)=w_{1} \otimes \tilde{\mathrm{F}}_{1} \oplus w_{2} \otimes \tilde{\mathrm{F}}_{2} \oplus w_{3} \otimes \tilde{\mathrm{F}}_{3}$ 


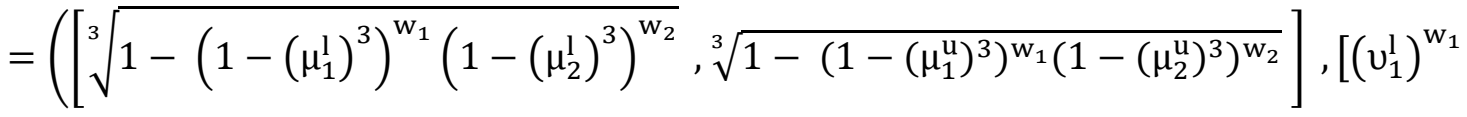

$$
\begin{aligned}
& \left.\left.\left.\times\left(v_{2}^{\mathrm{l}}\right)^{\mathrm{w}_{2}},\left(v_{1}^{\mathrm{u}}\right)^{\mathrm{w}_{1}} \times\left(v_{2}^{\mathrm{u}}\right)^{\mathrm{w}_{2}}\right]\right]\right) \\
& \oplus\left(\left[\sqrt[3]{1-\left(1-\left(\mu_{3}^{l}\right)^{3}\right)^{\mathrm{w}_{3}}}, \sqrt[3]{1-\left(1-\left(\mu_{3}^{\mathrm{u}}\right)^{3}\right)^{\mathrm{w}_{3}}}\right],\left[\left(v_{3}^{\mathrm{l}}\right)^{\mathrm{w}_{3}},\left(\mathrm{v}_{3}^{\mathrm{u}}\right)^{\mathrm{w}_{3}}\right]\right) \\
& =\left(\left[\begin{array}{l}
\sqrt[3]{1-\left(1-\left(\mu_{1}^{\mathrm{l}}\right)^{3}\right)^{\mathrm{w}_{1}}\left(1-\left(\mu_{2}^{\mathrm{l}}\right)^{3}\right)^{\mathrm{w}_{2}}+1-\left(1-\left(\mu_{3}^{\mathrm{l}}\right)^{3}\right)^{\mathrm{w}_{3}}-} \\
\left(1-\left(1-\left(\mu_{1}^{\mathrm{l}}\right)^{3}\right)^{\mathrm{w}_{1}}\left(1-\left(\mu_{2}^{\mathrm{l}}\right)^{3}\right)^{\mathrm{w}_{2}}\right)\left(1-\left(1-\left(\mu_{3}^{\mathrm{l}}\right)^{3}\right)^{\mathrm{w}_{3}}\right)^{\prime}
\end{array},\right.\right. \\
& \sqrt[3]{\begin{array}{c}
1-\left(1-\left(\mu_{1}^{\mathrm{u}}\right)^{3}\right)^{\mathrm{w}_{1}}\left(1-\left(\mu_{2}^{\mathrm{u}}\right)^{3}\right)^{\mathrm{w}_{2}}+1-\left(1-\left(\mu_{3}^{\mathrm{u}}\right)^{3}\right)^{\mathrm{w}_{3}}- \\
\left(1-\left(1-\left(\mu_{1}^{\mathrm{u}}\right)^{3}\right)^{\mathrm{w}_{1}}\left(1-\left(\mu_{2}^{\mathrm{u}}\right)^{3}\right)^{\mathrm{w}_{2}}\right)\left(1-\left(1-\left(\mu_{3}^{\mathrm{u}}\right)^{3}\right)^{\mathrm{w}_{3}}\right)
\end{array}},\left[\left(v_{1}^{\mathrm{l}}\right)^{\mathrm{w}_{1}} \times\left(v_{2}^{\mathrm{l}}\right)^{\mathrm{w}_{2}} \times\right. \\
& \left.\left.\left(v_{3}^{l}\right)^{w_{3}},\left(v_{1}^{u}\right)^{w_{1}} \times\left(v_{2}^{u}\right)^{w_{2}} \times\left(v_{3}^{u}\right)^{w_{3}}\right]\right) \\
& =\left(\left[\sqrt[3]{1-\left(1-\left(\mu_{1}^{1}\right)^{3}\right)^{w_{1}}\left(1-\left(\mu_{2}^{1}\right)^{3}\right)^{w_{2}}\left(1-\left(\mu_{3}^{1}\right)^{3}\right)^{w_{3}}},\right.\right. \\
& \left.\sqrt[3]{1-\left(1-\left(\mu_{1}^{\mathrm{u}}\right)^{3}\right)^{w_{1}}\left(1-\left(\mu_{2}^{u}\right)^{3}\right)^{w_{2}}\left(1-\left(\mu_{3}^{u}\right)^{3}\right)^{w_{3}}}\right],\left[\left(v_{1}^{\mathrm{l}}\right)^{w_{1}}\right. \\
& \left.\left.\left.\times\left(v_{2}^{\mathrm{l}}\right)^{w_{2}},\left(v_{1}^{\mathrm{u}}\right)^{w_{1}} \times\left(v_{2}^{\mathrm{u}}\right)^{w_{2}}\right]\right]\right) \\
& = \\
& \left(\left[\sqrt[3]{1-\prod_{\mathrm{k}=1}^{3}\left(1-\left(\mu_{\mathrm{k}}^{\mathrm{l}}\right)^{3}\right)^{\mathrm{w}_{\mathrm{k}}}}, \sqrt[3]{\left(1-\prod_{\mathrm{k}=1}^{3}\left(1-\left(\mu_{\mathrm{k}}^{\mathrm{u}}\right)^{3}\right)^{\mathrm{w}_{\mathrm{k}}}\right)}\right],\left[\left(\prod_{\mathrm{k}=1}^{3}\left(\mathrm{v}_{\mathrm{k}}^{\mathrm{l}}\right)^{\mathrm{w}_{\mathrm{k}}}\right),\left(\prod_{\mathrm{k}=1}^{3}\left(\mathrm{v}_{\mathrm{k}}^{\mathrm{u}}\right)^{\mathrm{w}_{\mathrm{k}}}\right)\right]\right)
\end{aligned}
$$

Similarly for N IVFFN's:

$\operatorname{IVFFWA}\left(\tilde{\mathrm{F}}_{1}, \tilde{\mathrm{F}}_{2}, \ldots \ldots, \tilde{\mathrm{F}}_{\mathrm{N}}\right)=w_{1} \otimes \tilde{\mathrm{F}}_{1} \oplus w_{2} \otimes \tilde{\mathrm{F}}_{2} \oplus \ldots \ldots \oplus w_{\mathrm{N}} \otimes \tilde{\mathrm{F}}_{\mathrm{N}}$

$\operatorname{IVFFWA}\left(\tilde{\mathrm{F}}_{1}, \tilde{\mathrm{F}}_{2}, \ldots \ldots, \tilde{\mathrm{F}}_{\mathrm{N}}\right)=\left(\left[\sqrt[3]{1-\prod_{\mathrm{k}=1}^{\mathrm{N}}\left(1-\left(\mu_{\mathrm{k}}^{\mathrm{l}}\right)^{3}\right)^{\mathrm{w}_{\mathrm{k}}}}, \sqrt[3]{\left(1-\prod_{\mathrm{k}=1}^{\mathrm{N}}\left(1-\left(\mu_{\mathrm{k}}^{\mathrm{u}}\right)^{3}\right)^{\mathrm{w}_{\mathrm{k}}}\right.}\right)\right]$

$\left.\left[\left(\prod_{\mathrm{k}=1}^{\mathrm{N}}\left(\mathrm{v}_{\mathrm{k}}^{\mathrm{l}}\right)^{\mathrm{w}_{\mathrm{k}}}\right),\left(\prod_{\mathrm{k}=1}^{\mathrm{N}}\left(\mathrm{v}_{\mathrm{k}}^{\mathrm{u}}\right)^{\mathrm{w}_{\mathrm{k}}}\right)\right]\right)$ 


\section{Proposed approach: MABAC model with IVFFNs}

The steps of proposed MABAC method with IVFFNs are described below.

Step-1: Construction of interval-valued fermatean fuzzy decision matrix. The alternatives are evaluated with respect to different criteria. $\tilde{\mathrm{x}}_{\mathrm{ij}}$ is the performance value of $\mathrm{i}^{\text {th }}$ alternative $(\mathrm{i}=1,2, \ldots, \mathrm{m})$ with respect to $\mathrm{j}^{\text {th }}$ criteria $(\mathrm{j}=1,2, \ldots, \mathrm{n})$ and $\mathrm{k}=1,2, \ldots, \mathrm{p}$ represent the number of decision-makers.

$\widetilde{\mathrm{X}}^{\mathrm{k}}=\left[\widetilde{\mathrm{X}}_{\mathrm{ij}}^{\mathrm{k}}\right]_{\mathrm{m} \times \mathrm{n}}$

$$
\begin{aligned}
& \begin{array}{llll}
\mathrm{C}_{1} & \mathrm{C}_{2} & \ldots & \mathrm{C}_{\mathrm{n}}
\end{array}
\end{aligned}
$$

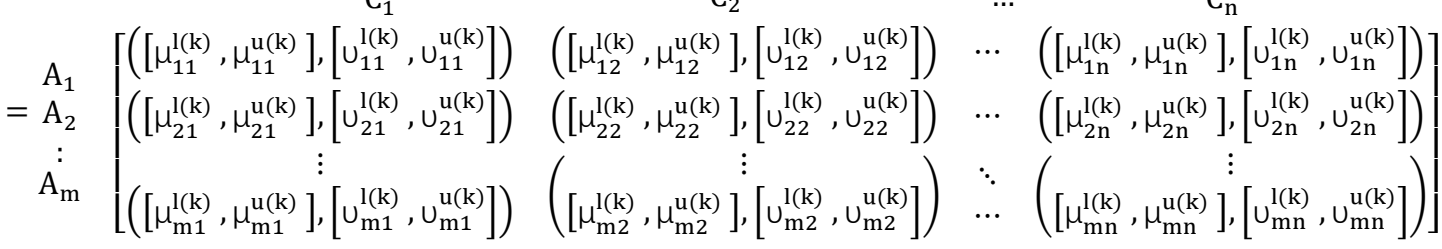

where $\mathrm{m}$ is the number of alternatives and $\mathrm{n}$ is the number of criteria.

Step-2: Normalize the interval-valued fermatean fuzzy decision matrix.

$\widetilde{\mathrm{N}}^{\mathrm{k}}=\left[\tilde{\mathrm{n}}_{\mathrm{ij}}^{\mathrm{k}}\right]_{\mathrm{m} \times \mathrm{n}}$

$\tilde{\mathrm{n}}_{\mathrm{ij}}^{\mathrm{k}}=\widetilde{\mathrm{X}}_{\mathrm{ij}}^{\mathrm{k}} \quad$ for beneficial criteria.

$\widetilde{\mathrm{n}}_{\mathrm{ij}}^{\mathrm{k}}=\left(\widetilde{\mathrm{X}}_{\mathrm{ij}}^{\mathrm{k}}\right)^{\mathrm{c}} \quad$ for non-beneficial criteria.

Step-3: Compute the weighted interval-valued fermatean fuzzy normalized matrix.

$$
\begin{aligned}
& \widetilde{\mathrm{V}}^{\mathrm{k}}=\left[\tilde{\mathrm{V}}_{\mathrm{ij}}^{\mathrm{k}}\right]_{\mathrm{m} \times \mathrm{n}}
\end{aligned}
$$

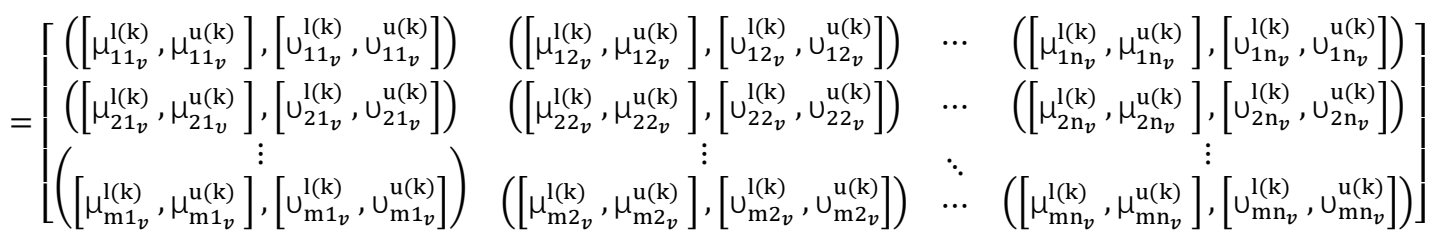

$$
\begin{aligned}
& \tilde{v}_{\mathrm{ij}}^{\mathrm{k}}=\mathrm{w}_{\mathrm{j}}^{\mathrm{k}} \tilde{\mathrm{n}}_{\mathrm{ij}}^{\mathrm{k}}=\left(\left[\sqrt[3]{1-\left(1-\left(\mu_{\mathrm{ij}}^{\mathrm{l}(\mathrm{k})}\right)^{3}\right)^{\mathrm{w}_{\mathrm{j}}^{\mathrm{k}}}}, \sqrt[3]{1-\left(1-\left(\mu_{\mathrm{ij}}^{\mathrm{u}(\mathrm{k})}\right)^{3}\right)^{\mathrm{w}_{\mathrm{j}}^{\mathrm{k}}}}\right],\left[\left(\mathrm{u}_{\mathrm{ij}}^{\mathrm{l}(\mathrm{k})}\right)^{\mathrm{w}_{\mathrm{j}}^{\mathrm{k}}},\left(\mathrm{u}_{\mathrm{ij}}^{\mathrm{u}(\mathrm{k})}\right)^{\mathrm{w}_{\mathrm{j}}^{\mathrm{k}}}\right]\right)= \\
& \left(\left[\mu_{\mathrm{ij}_{v}}^{\mathrm{l}(\mathrm{k})}, \mu_{\mathrm{ij}_{v}}^{\mathrm{u}(\mathrm{k})}\right],\left[\mathrm{U}_{\mathrm{ij}_{v}}^{\mathrm{l}(\mathrm{k})}, \mathrm{U}_{\mathrm{ij}_{v}}^{\mathrm{u}(\mathrm{k})}\right]\right)
\end{aligned}
$$

For calculating the weights of the criteria following equation can be used 
Let $\widetilde{w}_{j}^{k}=\left[\mu_{j}^{l(k)}, \mu_{j}^{u(k)}\right],\left[v_{j}^{l(k)}, v_{j}^{u(k)}\right]$ be the interval-valued fermatean fuzzy weights of the criteria.

$$
w_{j}^{k}=\frac{\mu_{j}^{l(k)}+\mu_{j}^{u(k)}+\left(\pi_{j}^{l(k)}+\pi_{j}^{u(k)}\right)\left(\frac{\mu_{j}^{l(k)}+\mu_{j}^{u(k)}}{\mu_{j}^{l(k)}+\mu_{j}^{u}(k)+v_{j}^{l(k)}+v_{j}^{u(k)}}\right)}{\sum_{j=1}^{n}\left(\mu_{j}^{l(k)}+\mu_{j}^{u(k)}+\left(\pi_{j}^{l(k)}+\pi_{j}^{u(k)}\right)\left(\frac{\mu_{j}^{l(k)}+\mu_{j}^{u(k)}}{\mu_{j}^{l(k)}+\mu_{j}^{u(k)}+v_{j}^{l(k)}+v_{j}^{u(k)}}\right)\right)}
$$

The aggregated weighted interval-valued fermatean fuzzy normalized matrix can be obtained by using the IVFFWA operator.

$\widetilde{V}=\operatorname{IVFFWA}\left(\widetilde{V}^{1}, \widetilde{V}^{2}, \ldots \ldots, \widetilde{V}^{p}\right)$, where $d_{1}, d_{2}, \ldots \ldots, d_{p}$ are the weights of the decision-makers.

$$
d_{k}=\frac{\mu^{l(k)}+\mu^{u(k)}+\left(\pi^{l(k)}+\pi^{u(k)}\right)\left(\frac{\mu^{l(k)}+\mu^{u}(k)}{\mu^{l(k)}+\mu^{u(k)}+v^{l(k)}+v^{u}(k)}\right)}{\sum_{k=1}^{p}\left(\mu^{l(k)}+\mu^{u(k)}+\left(\pi^{l(k)}+\pi^{u(k)}\right)\left(\frac{\mu^{1(k)}+\mu^{u(k)}}{\mu^{l(k)}+\mu^{u(k)}+v^{l(k)}+v^{u(k)}}\right)\right)} ; \text { where }\left[\mu^{l(k)}, \mu^{u(k)}\right],\left[v^{l(k)}, v^{u(k)}\right]
$$

be the interval-valued fermatean fuzzy weights of the $\mathrm{k}^{\text {th }}$ decision-maker.

Step-4: Compute the border approximation area (BAA) matrix $(\mathrm{G})$ for each criterion.

$$
\begin{aligned}
& \left.\widetilde{\mathrm{G}}=\left[\tilde{\mathrm{g}}_{i}\right]_{1 \times \mathrm{n}}=\begin{array}{cccc}
\mathrm{C}_{1} & \mathrm{C}_{2} & \ldots & \mathrm{C}_{\mathrm{n}} \\
\tilde{\mathrm{g}}_{1} & \tilde{\mathrm{g}}_{2} & \ldots & \tilde{\mathrm{g}}_{\mathrm{n}}
\end{array}\right] \\
& \tilde{\mathrm{g}}_{\mathrm{j}}=\left(\prod_{\mathrm{i}=1}^{\mathrm{m}} \tilde{\mathrm{V}}_{\mathrm{ij}}\right)^{1 / \mathrm{m}} \\
& = \\
& \left(\left[\left(\prod_{\mathrm{i}=1}^{\mathrm{m}} \mu_{\mathrm{ij}_{v}}^{\mathrm{l}}\right)^{1 / \mathrm{m}},\left(\prod_{\mathrm{i}=1}^{\mathrm{m}} \mu_{\mathrm{ij}}^{\mathrm{u}}\right)^{1 / \mathrm{m}}\right],\left[\sqrt[3]{1-\left(\prod_{\mathrm{i}=1}^{\mathrm{m}}\left(1-\left(\mathrm{u}_{\mathrm{ij}}^{\mathrm{l}}\right)^{3}\right)\right)^{1 / \mathrm{m}}}, \sqrt[3]{1-\left(\prod_{\mathrm{i}=1}^{\mathrm{m}}\left(1-\left(\mathrm{u}_{\mathrm{ij} v}^{\mathrm{u}}\right)^{3}\right)\right)^{1 / m}}\right]\right)
\end{aligned}
$$

Step-5: Compute the distance of the alternative from the BAA- The distance of the alternatives from the BAA is computed using IVFFHD. As the Hamming distance calculates the absolute value, to know $\tilde{v}_{i j}>\tilde{g}_{j}$ or $\tilde{v}_{i j}=\tilde{g}_{j}$ or $\tilde{v}_{i j}<\tilde{g}_{j}$, we will use the score function: 


$$
\begin{aligned}
& \mathrm{D}=\left[\mathrm{d}_{\mathrm{ij}}\right]_{\mathrm{m} \times \mathrm{n}}=\begin{array}{c}
\mathrm{C}_{1} \\
\mathrm{~A}_{1} \\
\mathrm{~A}_{2} \\
\vdots \\
\mathrm{A}_{\mathrm{m}}
\end{array}\left[\begin{array}{cccc}
\mathrm{d}_{11} & \mathrm{~d}_{12} & \cdots & \mathrm{C}_{1 \mathrm{n}} \\
\mathrm{d}_{21} & \mathrm{~d}_{22} & \cdots & \mathrm{d}_{2 \mathrm{n}} \\
\vdots & \vdots & \ddots & \vdots \\
\mathrm{d}_{\mathrm{m} 1} & \mathrm{~d}_{\mathrm{m} 2} & \cdots & \mathrm{d}_{\mathrm{mn}}
\end{array}\right] \\
& \begin{array}{cccc}
\mathrm{C}_{1} & \mathrm{C}_{2} & \ldots & \mathrm{C}_{\mathrm{n}}
\end{array} \\
& \mathrm{A}_{1} \quad\left[\begin{array}{llll}
d_{I V F F H D}\left(\tilde{\mathrm{v}}_{11}, \tilde{\mathrm{g}}_{1}\right) \quad d_{I V F F H D}\left(\tilde{\mathrm{v}}_{12}, \tilde{\mathrm{g}}_{2}\right) & \cdots & d_{I V F F H D}\left(\tilde{\mathrm{v}}_{1 \mathrm{n}}, \tilde{\mathrm{g}}_{\mathrm{n}}\right)
\end{array}\right]
\end{aligned}
$$

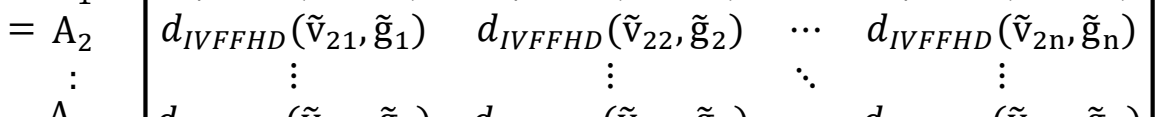

$$
\begin{aligned}
& \left.\mathrm{A}_{\mathrm{m}} \quad d_{I V F F H D}\left(\tilde{\mathrm{v}}_{\mathrm{m} 1}, \tilde{\mathrm{g}}_{1}\right) \quad d_{I V F F H D}\left(\tilde{\mathrm{v}}_{\mathrm{m} 2}, \tilde{\mathrm{g}}_{2}\right) \quad \cdots \quad d_{I V F F H D}\left(\tilde{\mathrm{v}}_{\mathrm{mn}}, \tilde{\mathrm{g}}_{\mathrm{n}}\right)\right] \\
& d_{i j}= \begin{cases}-d_{\text {IVFFHD }}\left(\tilde{v}_{11}, \tilde{g}_{1}\right) & \text { if } \tilde{v}_{i j}>\tilde{g}_{j} \\
0 & \text { if } \tilde{v}_{i j}=\tilde{g}_{j} \\
d_{\text {IVFFHD }}\left(\tilde{v}_{11}, \tilde{g}_{1}\right) & \text { if } \tilde{v}_{i j}<\tilde{g}_{j}\end{cases}
\end{aligned}
$$

Step-6: Ranking the alternatives. The alternatives are ranked based on the comprehensive evaluation result $\left(S_{i}\right)$ which is computed by summing the distance values of each alternative. The larger the value of $S_{i}$, the better is the alternative.

$$
\mathrm{S}_{\mathrm{i}}=\sum_{j=1}^{n} \mathrm{~d}_{\mathrm{ij}}
$$

\section{MODEL IMPLEMENTATION}

While selecting a potential green supplier, both traditional and environmental criteria should be considered. Traditional criteria include: desired quality, purchase cost, procurement costs, on-time delivery, capability to adapt emerging technology, trust, and flexibility. Environmental criteria include sustainability in procurement procedures, recyclable packaging, use of environmental products, environmental, social responsibilities, reduced carbon emission, lower energy and water consumption, conformance to environmental policies, and having certifications such as ISO 14000 and ISO 14001. To implement and validate the proposed model, a green supplier selection problem from the work of Rouyendegh et al. (2020) is solved using the interval-valued fermatean fuzzy MABAC. A 
company located in Ankara, Turkey wants to select the best green supplier among four alternatives based on ten criteria: Quality (C1), Cost (C2), Service and Delivery (C3), Sustainability (C4), Technology (C5), Green Manufacturing System (C6), Green Supplier Image (C7), Cooperation (C8), Green Application (C9) and Environmental Management and Control (C10). There are three decision-makers, and the importance is determined using linguistic terms scale shown in Table-1. The importance of the three decision-makers are "very important", "important", and "medium" respectively. The IVFFN corresponding to linguistic terms are used to compute the importance of decision-makers. The importance levels of decision-makers given in linguistic terms are converted to crisp numerical values using equation 2 such that the importance levels of the three decision-makers are 0.3893 , 0.3443 and 0.2664 . Next, the importance of evaluating criteria (weights of criteria) is determined using linguistic terms scale (Table-1) which is shown in Table-3. The IVFFN corresponding to linguistic terms are used to compute the importance of evaluating criteria. The importance of criteria given in linguistic terms is converted to crisp numerical values using equation 2 .

Table-1 Linguistic terms scale for weights of criteria

\begin{tabular}{|c|c|}
\hline Linguistic terms & IVFFN \\
\hline Very important (VI) & $([0.8,0.9],[0.1,0.2])$ \\
\hline Important (I) & $([0.7,0.8],[0.2,0.5])$ \\
\hline Medium (M) & $([0.5,0.7],[0.5,0.7])$ \\
\hline Unimportant (U) & $([0.2,0.5],[0.7,0.8])$ \\
\hline Very unimportant (VU) & $([0.1,0.2],[0.8,0.9])$ \\
\hline
\end{tabular}

Table-2 Linguistic terms scale for rating the alternatives

\begin{tabular}{|c|c|}
\hline Linguistic terms & IVFFN \\
\hline Very poor (VP) & $([0.2,0.3],[0.8,0.9])$ \\
\hline Poor $(\mathrm{P})$ & $([0.3,0.4],[0.7,0.8])$ \\
\hline Medium poor (MP) & $([0.4,0.5],[0.6,0.7])$ \\
\hline
\end{tabular}




\begin{tabular}{|c|c|}
\hline Fair $(\mathrm{F})$ & $([0.5,0.6],[0.5,0.6])$ \\
\hline Medium good $(\mathrm{MG})$ & $([0.6,0.7],[0.4,0.5])$ \\
\hline Good $(\mathrm{G})$ & $([0.7,0.8],[0.3,0.4])$ \\
\hline Very good $(\mathrm{VG})$ & $([0.8,0.9],[0.2,0.3])$ \\
\hline
\end{tabular}

Table-3 Importance of criteria based on the opinion of decision-makers

\begin{tabular}{|l|c|c|c|c|c|c|c|c|c|c|}
\hline & C1 & C2 & C3 & C4 & C5 & C6 & C7 & C8 & C9 & C10 \\
\hline $\begin{array}{l}\text { Decision- } \\
\text { maker-1 }\end{array}$ & VI & I & M & I & M & I & M & U & I & I \\
\hline $\begin{array}{l}\text { Decision- } \\
\text { maker-2 }\end{array}$ & I & M & M & VI & U & I & I & U & VI & I \\
\hline $\begin{array}{l}\text { Decision- } \\
\text { maker-3 }\end{array}$ & I & I & U & I & VI & VI & U & M & M & I \\
\hline
\end{tabular}

The performance value of each alternative with respect to the evaluation criteria is shown in Table-4. The linguistic terms scale shown in Table- 2 is used to convert the linguistic term to IVFFN and formulate the interval-valued fermatean fuzzy decision matrix. The intervalvalued fermatean fuzzy decision matrix is normalized based on the nature of the criterion (beneficial or non-beneficial). In the problem Cost (C2) is non-beneficial and the remaining nine criteria are beneficial in nature. The weighted interval-valued fermatean fuzzy normalized matrix for all three decision-makers is computed by multiplying the weights of criteria with the normalized interval-valued fermatean fuzzy decision matrix using equation1. The graphical representation of criteria weights is shown in Figure 2. 


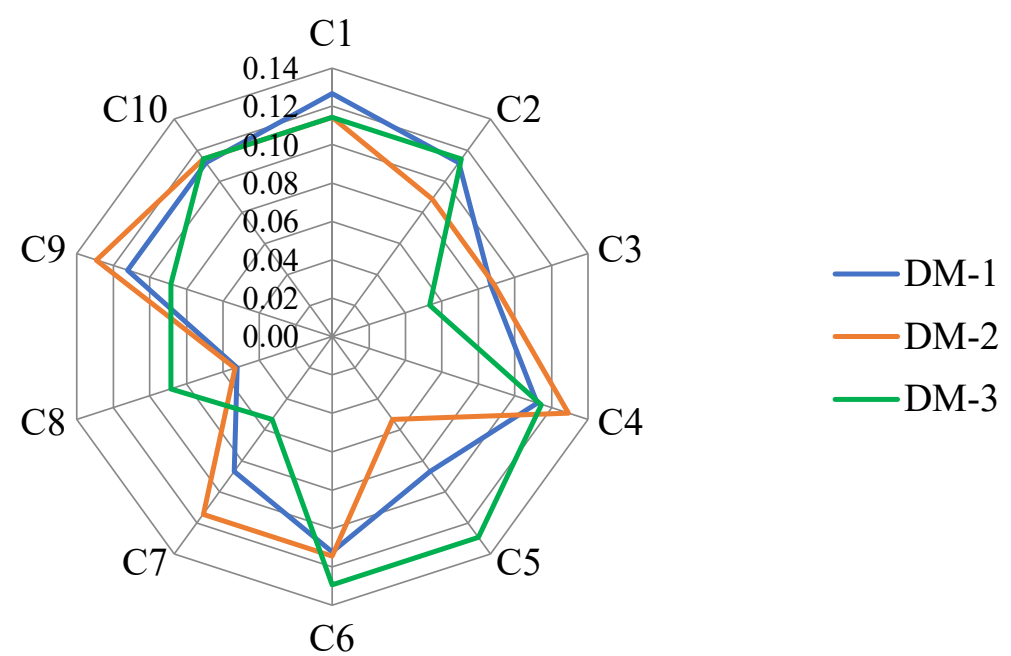

Figure 2 Relative importance of criteria weights

Table-4 Performance value of alternatives based on criteria

\begin{tabular}{|c|c|c|c|c|c|c|c|c|c|c|c|}
\hline & C1 & C2 & C3 & C4 & C5 & C6 & C7 & C8 & C9 & C10 \\
\hline & \multicolumn{7}{|c|}{ Decision-maker 1 } \\
\hline A1 & G & G & MG & MG & F & MG & F & VG & MG & G \\
\hline A2 & G & VG & G & G & G & G & MG & G & G & VG \\
\hline A3 & MG & MG & MG & F & MP & P & P & G & VP & G \\
\hline A4 & VG & G & G & VG & G & VG & G & VG & VG & VG \\
\hline & \multicolumn{7}{|c|}{ Decision-maker 2 } \\
\hline A1 & MG & G & G & F & MG & G & MG & G & F & G \\
\hline A2 & VG & G & G & VG & G & G & G & VG & MG & G \\
\hline A3 & G & MG & F & P & MP & F & F & G & F & G \\
\hline A4 & VG & VG & G & VG & VG & G & G & MG & G & VG \\
\hline & \multicolumn{7}{|c|}{} & \multicolumn{7}{|c|}{ Decision-maker 3 } & & \\
\hline A1 & MG & G & G & MG & MG & F & F & VG & G & VG \\
\hline A2 & G & VG & G & G & VG & G & G & MG & G & VG \\
\hline A3 & MG & MG & MP & F & P & MP & MG & VG & F & G \\
\hline A4 & VG & VG & MG & G & VG & G & VG & G & VG & VG \\
\hline
\end{tabular}

Next, the aggregated normalized interval-valued fermatean fuzzy decision matrix is computed - as shown in Table-5 which has the border approximation area (BAA) matrix (G) for each criterion computed using equation 3. The computed distances $\left(d_{i j}\right)$ between alternatives and BAA is shown in Table-6. To determine $\tilde{v}_{i j}>\tilde{g}_{j}$ or $\tilde{v}_{i j}=\tilde{g}_{j}$ or $\tilde{v}_{i j}<\tilde{g}_{j}$, the 
score function is used. Once the distances $\left(\mathrm{d}_{\mathrm{ij}}\right)$ between alternatives and BAA is computed, the comprehensive evaluation result $\left(S_{\mathrm{i}}\right)$ is calculated by summing the distance values of each alternative. The comprehensive evaluation result along with its corresponding rank is shown in Table7.

Table-5 Aggregated weighted interval-valued fermatean fuzzy normalized matrix

\begin{tabular}{|c|c|c|c|c|c|}
\hline & C1 & $\mathrm{C2}$ & $\mathrm{C3}$ & $\mathrm{C4}$ & C5 \\
\hline A1 & $\begin{array}{l}([0.333,0.397], \\
[0.884,0.911])\end{array}$ & $\begin{array}{c}([0.142,0.190], \\
[0.963,0.977])\end{array}$ & $\begin{array}{c}([0.299,0.356], \\
[0.919,0.938])\end{array}$ & $\begin{array}{c}([0.287,0.345], \\
[0.906,0.929])\end{array}$ & $\begin{array}{c}([0.258,0.311], \\
[0.931,0.948])\end{array}$ \\
\hline A2 & $\begin{array}{l}([0.391,0.469], \\
[0.853,0.886])\end{array}$ & $\begin{array}{c}([0.113,0.159], \\
[0.973,0.985])\end{array}$ & $\begin{array}{c}([0.319,0.380], \\
[0.910,0.931])\end{array}$ & $\begin{array}{c}([0.394,0.472], \\
[0.851,0.886])\end{array}$ & $\begin{array}{l}([0.357,0.429], \\
[0.888,0.915])\end{array}$ \\
\hline A3 & $\begin{array}{l}([0.328,0.391], \\
[0.886,0.913])\end{array}$ & $\begin{array}{c}([0.190,0.240], \\
[0.948,0.963])\end{array}$ & $\begin{array}{c}([0.236,0.285], \\
[0.942,0.957])\end{array}$ & $\begin{array}{l}([0.223,0.275], \\
[0.935,0.953])\end{array}$ & $\begin{array}{l}([0.164,0.210], \\
[0.962,0.974])\end{array}$ \\
\hline A4 & $\begin{array}{l}([0.434,0.524], \\
[0.825,0.866])\end{array}$ & $\begin{array}{l}([0.119,0.166], \\
[0.971,0.984])\end{array}$ & $\begin{array}{l}([0.311,0.370], \\
[0.914,0.934])\end{array}$ & $\begin{array}{l}([0.418,0.504], \\
[0.836,0.875])\end{array}$ & $\begin{array}{l}([0.370,0.446], \\
[0.882,0.910])\end{array}$ \\
\hline$\tilde{\mathrm{g}}_{\mathrm{j}}$ & $\begin{array}{l}([0.369,0.442], \\
[0.865,0.896])\end{array}$ & $\begin{array}{l}([0.138,0.186], \\
[0.965,0.979])\end{array}$ & $\begin{array}{l}([0.289,0.346], \\
[0.922,0.941])\end{array}$ & $\begin{array}{l}([0.320,0.388], \\
[0.891,0.917])\end{array}$ & $\begin{array}{l}([0.273,0.334], \\
[0.924,0.944])\end{array}$ \\
\hline & C6 & C & $\mathrm{Ce}$ & $\mathbf{C}$ & 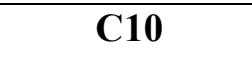 \\
\hline A1 & $\begin{array}{l}([0.315,0.377], \\
[0.895,0.919])\end{array}$ & $\begin{array}{l}([0.251,0.303], \\
[0.933,0.950])\end{array}$ & $\begin{array}{l}([0.338,0.408], \\
[0.911,0.933])\end{array}$ & $\begin{array}{l}([0.297,0.356], \\
[0.906,0.928])\end{array}$ & $\begin{array}{l}([0.381,0.456], \\
[0.862,0.893])\end{array}$ \\
\hline A2 & $\begin{array}{l}([0.364,0.432], \\
[0.868,0.898])\end{array}$ & $\begin{array}{l}([0.312,0.372], \\
[0.909,0.930])\end{array}$ & $\begin{array}{l}([0.300,0.362], \\
[0.927,0.944])\end{array}$ & $\begin{array}{c}([0.337,0.402], \\
[0.885,0.912])\end{array}$ & $\begin{array}{c}([0.407,0.490], \\
[0.846,0.882])\end{array}$ \\
\hline A3 & $\begin{array}{l}([0.206,0.257], \\
[0.941,0.959])\end{array}$ & $\begin{array}{l}([0.213,0.260], \\
[0.949,0.963])\end{array}$ & $\begin{array}{l}([0.320,0.385], \\
[0.919,0.938])\end{array}$ & $\begin{array}{l}([0.211,0.260], \\
[0.945,0.961])\end{array}$ & $\begin{array}{l}([0.360,0.428], \\
[0.872,0.901])\end{array}$ \\
\hline A4 & $\begin{array}{l}([0.392,0.470], \\
[0.853,0.887])\end{array}$ & $\begin{array}{c}([0.342,0.409], \\
[0.895,0.919])\end{array}$ & $\begin{array}{c}([0.306,0.368], \\
[0.925,0.943])\end{array}$ & $\begin{array}{c}([0.402,0.483], \\
[0.851,0.885])\end{array}$ & $\begin{array}{c}([0.428,0.516], \\
[0.833,0.872])\end{array}$ \\
\hline$\tilde{\mathrm{g}}_{\mathrm{j}}$ & $\begin{array}{l}([0.310,0.375], \\
[0.896,0.922])\end{array}$ & $\begin{array}{l}([0.275,0.331], \\
[0.925,0.943])\end{array}$ & $\begin{array}{l}([0.316,0.380], \\
[0.921,0.940])\end{array}$ & $\begin{array}{l}([0.303,0.366], \\
[0.903,0.928])\end{array}$ & $\begin{array}{l}([0.393,0.471], \\
[0.854,0.888])\end{array}$ \\
\hline
\end{tabular}

Table-6 The distances $d_{i j}$ between alternatives and BAA

\begin{tabular}{|c|c|c|c|c|c|c|c|c|c|c|}
\hline & C1 & C2 & C3 & C4 & C5 & C6 & C7 & C8 & C9 & C10 \\
\hline A1 & -0.040 & 0.005 & 0.009 & -0.033 & -0.014 & 0.005 & -0.019 & 0.021 & -0.005 & $\begin{array}{c}- \\
0.015\end{array}$ \\
\hline $\mathbf{A 2}$ & 0.025 & -0.020 & 0.029 & 0.083 & 0.081 & 0.062 & 0.037 & -0.014 & 0.042 & 0.015 \\
\hline
\end{tabular}




\begin{tabular}{|l|c|c|c|c|c|c|c|c|c|c|}
\hline $\mathbf{A 3}$ & -0.046 & 0.046 & -0.048 & -0.103 & -0.093 & -0.106 & -0.059 & 0.005 & -0.098 & - \\
\hline $\mathbf{A 4}$ & 0.076 & -0.016 & 0.021 & 0.112 & 0.095 & 0.093 & 0.068 & -0.009 & 0.113 & 0.041 \\
\hline
\end{tabular}

Table-7 Comprehensive evaluation result along with the rank of alternatives

\begin{tabular}{|c|c|c|}
\hline & Si & Rank \\
\hline $\mathbf{A 1}$ & -0.086 & 3 \\
\hline $\mathbf{A 2}$ & 0.340 & 2 \\
\hline $\mathbf{A 3}$ & -0.538 & 4 \\
\hline $\mathbf{A 4}$ & 0.593 & 1 \\
\hline
\end{tabular}

\section{Result and discussion}

From the comprehensive evaluation result and ranking order of alternative it can be seen that alternative-4 (A4) is the best green supplier with the Si value of 0.593 and the next best alternative is alternative-2 (A2) with the $\mathrm{Si}$ value of 0.340 . The worst alternative is alternative-3 (A3) with the Si value of -0.538 . The ranking order obtained is compared with the ranking order obtained from other researchers in literature. To check the stability and robustness of the proposed method, a sensitivity analysis is also performed.

\subsection{Comparative analysis}

The comparison of rank obtained from interval-valued fermatean fuzzy MABAC is completed with Intuitionistic Fuzzy TOPSIS (Rouyendegh et al., 2020) in Table 8. Rouyendegh et al. (2020) solved the green supplier selection problem with Intuitionistic Fuzzy TOPSIS and alternative 2 was assessed as the best choice with rank 1, whereas on solving the same problem with interval-valued fermatean fuzzy MABAC, alternative 4 is computed as the best alternative with rank 1 . The five points linguistic terms scale for weights of criteria and seven points linguistic terms scale for rating the alternatives with intuitionistic fuzzy numbers were used by Rouyendegh et al. (2020). To ensure the work was comparable, a similar five points linguistic terms scale for weights of criteria and seven points linguistic terms scale for rating the alternatives with interval-valued fermatean fuzzy is 
used in this work. Alternative 1 and Alternative 3 have same rank in both the methods. The ranks obtained by Rouyendegh et al. (2020) were not validated using sensitivity analysis. So, to validate the rank obtained by IVFF-MABAC, we performed a sensitivity analysis.

Table-8 Comparison of ranking using different methods

\begin{tabular}{|c|c|c|}
\hline & $\begin{array}{l}\text { Intuitionistic Fuzzy TOPSIS } \\
\text { (Rouyendegh et al., 2020) Rank }\end{array}$ & $\begin{array}{c}\text { IVF-MABAC } \\
\text { Rank }\end{array}$ \\
\hline A1 & 3 & 3 \\
\hline A2 & 1 & 2 \\
\hline A3 & 4 & 4 \\
\hline A4 & 2 & 1 \\
\hline
\end{tabular}

\subsection{Sensitivity Analysis}

The final ranking order of green supplier depends on two variable (the importance of decision-maker and evaluation of criteria weights), thus to check the stability and robustness of the obtained solutions a sensitivity analysis was performed by varying the importance of decision-makers and changing the weights of evaluation criteria.

The first analysis is done by assigning $100 \%$ weight to a single decision-maker. As there are three decision-makers, three different sets of comprehensive evaluation results and rankings are obtained, which is shown in Table 9. It can be observed that the ranking results for all three decision-makers are the same and alternative-4 (A4) is computed as the best green supplier.

Table 9 Ranking results of three decision-makers

\begin{tabular}{|l|l|l|l|l|l|}
\hline $\begin{array}{l}\mathbf{1 0 0 \%} \\
\text { Decision-maker-1 }\end{array}$ & \multicolumn{2}{|l|}{$\begin{array}{l}\mathbf{1 0 0 \%} \text { wecishtan-mentage } \\
\text { Decisiontager-2 }\end{array}$} & $\begin{array}{l}\mathbf{1 0 0 \%} \text { weightage to } \\
\text { Decision-maker-3 }\end{array}$ \\
\hline Si & Rank & Si & Rank & Si & Rank \\
\hline-0.033 & 3 & -0.133 & 3 & 0.008 & 3 \\
\hline 0.340 & 2 & 0.423 & 2 & 0.315 & 2 \\
\hline-0.594 & 4 & -0.460 & 4 & -0.458 & 4 \\
\hline 0.786 & 1 & 0.502 & 1 & 0.521 & 1 \\
\hline
\end{tabular}


In the second analysis, equal importance is given to each decision-maker and the weights of each criterion are increased by $20 \%$ in every set. As there are 10 criteria, 10 different set of weights are used in the sensitivity analysis. The comprehensive evaluation results along with their corresponding ranking are shown in Table 10 from which can be observed that the order of the alternatives remains the same in all 10 sets of weights and alternative-4 (A4) remains the best green supplier. So, the method IVFF-MABAC is stable and robust. It can be noted that.

Table 10 Sensitivity analysis by changing the weights of criteria

\begin{tabular}{|c|c|c|c|c|c|c|c|c|c|}
\hline \multicolumn{2}{|l|}{ set-1 } & \multicolumn{2}{|l|}{ set-2 } & \multicolumn{2}{|l|}{ set-3 } & \multicolumn{2}{|l|}{ set-4 } & \multicolumn{2}{|l|}{ set-5 } \\
\hline Si & Rank & $\mathbf{S i}$ & Rank & Si & Rank & $\mathbf{S i}$ & Rank & $\mathbf{S i}$ & Rank \\
\hline-0.096 & 3 & -0.060 & 3 & -0.045 & 3 & -0.087 & 3 & -0.081 & 3 \\
\hline 0.302 & 2 & 0.253 & 2 & 0.328 & 2 & 0.338 & 2 & 0.365 & 2 \\
\hline-0.462 & 4 & -0.366 & 4 & -0.518 & 4 & -0.523 & 4 & -0.567 & 4 \\
\hline 0.538 & 1 & 0.460 & 1 & 0.514 & 1 & 0.556 & 1 & 0.593 & 1 \\
\hline \multicolumn{2}{|c|}{ set-6 } & \multicolumn{2}{|c|}{ set-7 } & \multicolumn{2}{|c|}{ set-8 } & \multicolumn{2}{|c|}{ set-9 } & \multicolumn{2}{|c|}{ set-10 } \\
\hline $\mathbf{S i}$ & Rank & $\mathbf{S i}$ & Rank & $\mathbf{S i}$ & Rank & $\mathbf{S i}$ & Rank & $\mathbf{S i}$ & Rank \\
\hline-0.062 & 3 & -0.110 & 3 & -0.046 & 3 & -0.058 & 3 & -0.076 & 3 \\
\hline 0.333 & 2 & 0.327 & 2 & 0.274 & 2 & 0.322 & 2 & 0.296 & 2 \\
\hline-0.536 & 4 & -0.508 & 4 & -0.438 & 4 & -0.538 & 4 & -0.463 & 4 \\
\hline 0.555 & 1 & 0.576 & 1 & 0.478 & 1 & 0.570 & 1 & 0.517 & 1 \\
\hline
\end{tabular}

\section{Conclusion}

This article proposes an interval-valued fermatean fuzzy set and illustrates the fundamental set and arithmetic operation on IVFFS's along with its corresponding properties. The Hamming distance function and the score function of the interval-valued fermatean fuzzy numbers are also proposed. An interval-valued fermatean fuzzy MABAC method is proposed and the steps used in the method are explained. The distance of the alternatives from the BAA is computed using the interval-valued fermatean fuzzy Hamming distance. As the green supplier selection problem involves subjective criteria and uncertainty in preferences, we considered a numerical problem of green supplier selection and solved it using the proposed interval-valued fermatean fuzzy MABAC method. A sensitivity analysis was performed to 
determine the effect of the relative importance of decision-makers and evaluation criteria weights. The proposed method is shown to be stable and robust. Therefore, the proposed IVFF-MABAC can handle greater uncertainty than intuitionistic fuzzy and Pythagorean fuzzy MCDM methods.

Future research can use other recently developed MCDM techniques with the proposed interval-valued fermatean fuzzy set to obtain better results. The proposed intervalvalued fermatean fuzzy MABAC can also be applied to additional engineering and management applications.

\section{Reference}

Ashtiani, B., Haghighirad, F., Makui, A., \& ali Montazer, G. (2009). Extension of fuzzy TOPSIS method based on interval-valued fuzzy sets. Applied Soft Computing, 9(2), 457-461.

Atanassov, K. (1986). Intuitionistic fuzzy sets. Fuzzy sets and systems, 20, 87-96.

Brauers, W. K., \& Zavadskas, E. K. (2006). The MOORA method and its application to privatization in a transition economy. Control and cybernetics, 35, 445-469.

Çalık, A. (2021). A novel Pythagorean fuzzy AHP and fuzzy TOPSIS methodology for green supplier selection in the Industry 4.0 era. Soft Computing, 25(3), 2253-2265.

Fan, J., Liu, X., Wu, M., \& Wang, Z. (2019). Green supplier selection with undesirable outputs DEA under Pythagorean fuzzy environment. Journal of Intelligent \& Fuzzy Systems, 37(2), 24432452.

Fishburn, P. C. (1967). Letter to the editor-additive utilities with incomplete product sets: application to priorities and assignments. Operations Research, 15(3), 537-542.

Gitinavard, H., Ghaderi, H., \& Pishvaee, M. S. (2018). Green supplier evaluation in manufacturing systems: a novel interval-valued hesitant fuzzy group outranking approach. Soft Computing, 22(19), 6441-6460.

Grattan-Guinness, I. (1976). Fuzzy membership mapped onto intervals and many-valued quantities. Mathematical Logic Quarterly, 22(1), 149-160. 
Hwang, C.-L., \& Yoon, K. (1981). Methods for multiple attribute decision-making. In Multiple attribute decision-making (pp. 58-191): Springer.

Jahn, K. U. (1975). Intervall-wertige Mengen. Mathematische Nachrichten, 68(1), 115-132.

Jia, F., Liu, Y., \& Wang, X. (2019). An extended MABAC method for multi-criteria group decisionmaking based on intuitionistic fuzzy rough numbers. Expert Systems with Applications, 127, 241-255.

Kahraman, C., Öztayşi, B., \& Çevik Onar, S. (2016). A comprehensive literature review of 50 years of fuzzy set theory. International Journal of Computational intelligence systems, 9(sup1), 324.

Krishankumar, R., Gowtham, Y., Ahmed, I., Ravichandran, K., \& Kar, S. (2020). Solving green supplier selection problem using q-rung orthopair fuzzy-based decision framework with unknown weight information. Applied Soft Computing, 94, 106431.

Kumar, A., Jain, V., \& Kumar, S. (2014). A comprehensive environment friendly approach for supplier selection. Omega, 42(1), 109-123.

Kumari, R., \& Mishra, A. R. (2020). Multi-criteria COPRAS method based on parametric measures for intuitionistic fuzzy sets: application of green supplier selection. Iranian Journal of Science and Technology, Transactions of Electrical Engineering, 44(4), 1645-1662.

Liang, R.-x., He, S.-s., Wang, J.-q., Chen, K., \& Li, L. (2019). An extended MABAC method for multi-criteria group decision-making problems based on correlative inputs of intuitionistic fuzzy information. Computational and Applied Mathematics, 38(3), 1-28.

Liu, D., Liu, Y., \& Chen, X. (2019a). Fermatean fuzzy linguistic set and its application in multicriteria decision-making. International Journal of Intelligent Systems, 34(5), 878-894.

Liu, L., Cao, W., Shi, B., \& Tang, M. (2019b). Large-scale green supplier selection approach under a q-rung interval-valued orthopair fuzzy environment. Processes, 7(9), 573.

Mathew, M., Chakrabortty, R. K., \& Ryan, M. J. (2020a). A novel approach integrating AHP and TOPSIS under spherical fuzzy sets for advanced manufacturing system selection. Engineering Applications of Artificial Intelligence, 96, 103988. doi:10.1016/j.engappai.2020.103988 
Mathew, M., Chakrabortty, R. K., \& Ryan, M. J. (2020b). Selection of an Optimal Maintenance Strategy Under Uncertain Conditions: An Interval Type-2 Fuzzy AHP-TOPSIS Method. IEEE Transactions on Engineering Management, 1 - 14. doi:10.1109/TEM.2020.2977141

Mishra, A. R., Chandel, A., \& Motwani, D. (2020a). Extended MABAC method based on divergence measures for multi-criteria assessment of programming language with interval-valued intuitionistic fuzzy sets. Granular Computing, 5(1), 97-117.

Mishra, A. R., Garg, A. K., Purwar, H., Rana, P., Liao, H., \& Mardani, A. (2020b). An extended intuitionistic fuzzy multi-attributive border approximation area comparison approach for smartphone selection using discrimination measures. Informatica, 1-25.

Opricovic, S. (1998). Multicriteria optimization of civil engineering systems. Faculty of Civil Engineering, Belgrade, 2(1), 5-21.

Pamučar, D., \& Ćirović, G. (2015). The selection of transport and handling resources in logistics centers using Multi-Attributive Border Approximation area Comparison (MABAC). Expert Systems with Applications, 42(6), 3016-3028.

Peng, X., \& Dai, J. (2018). Approaches to single-valued neutrosophic MADM based on MABAC, TOPSIS and new similarity measure with score function. Neural Computing and Applications, 29(10), 939-954.

Peng, X., \& Yang, Y. (2016). Pythagorean fuzzy Choquet integral based MABAC method for multiple attribute group decision-making. International Journal of Intelligent Systems, 31(10), 989-1020.

Rouyendegh, B. D., Yildizbasi, A., \& Üstünyer, P. (2020). Intuitionistic fuzzy TOPSIS method for green supplier selection problem. Soft Computing, 24(3), 2215-2228.

Senapati, T., \& Yager, R. R. (2019). Some new operations over Fermatean fuzzy numbers and application of Fermatean fuzzy WPM in multiple criteria decision-making. Informatica, $30(2), 391-412$.

Senapati, T., \& Yager, R. R. (2020). Fermatean fuzzy sets. Journal of Ambient Intelligence and Humanized Computing, 11(2), 663-674. 
Shi, H., Quan, M.-Y., Liu, H.-C., \& Duan, C.-Y. (2018). A novel integrated approach for green supplier selection with interval-valued intuitionistic uncertain linguistic information: A case study in the agri-food industry. Sustainability, 10(3), 733.

Tian, Z.-P., Zhang, H.-Y., Wang, J.-Q., \& Wang, T.-L. (2018). Green supplier selection using improved TOPSIS and best-worst method under intuitionistic fuzzy environment. Informatica, 29(4), 773-800.

Wang, Wei, G., Wei, C., \& Wei, Y. (2020). MABAC method for multiple attribute group decisionmaking under q-rung orthopair fuzzy environment. Defence Technology, 16(1), 208-216.

Wang, J., Gao, H., Wei, G., \& Wei, Y. (2019). Methods for multiple-attribute group decision-making with q-rung interval-valued orthopair fuzzy information and their applications to the selection of green suppliers. Symmetry, 11(1), 56 .

Wang, R., \& Li, Y. (2018). A novel approach for green supplier selection under a q-rung orthopair fuzzy environment. Symmetry, 10(12), 687.

Wu, M.-Q., Zhang, C.-H., Liu, X.-N., \& Fan, J.-P. (2019). Green supplier selection based on DEA model in interval-valued Pythagorean fuzzy environment. Ieee Access, 7, 108001-108013.

Xiao, L., Zhang, S., Wei, G., Wu, J., Wei, C., Guo, Y., \& Wei, Y. (2020). Green supplier selection in steel industry with intuitionistic fuzzy Taxonomy method. Journal of Intelligent \& Fuzzy Systems(Preprint), 1-12.

Xue, Y.-X., You, J.-X., Lai, X.-D., \& Liu, H.-C. (2016). An interval-valued intuitionistic fuzzy MABAC approach for material selection with incomplete weight information. Applied Soft Computing, 38, 703-713.

Yager, R. R. (2013). Pythagorean membership grades in multi-criteria decision-making. IEEE Transactions on Fuzzy systems, 22(4), 958-965.

Yager, R. R. (2016). Generalized orthopair fuzzy sets. IEEE Transactions on Fuzzy systems, 25(5), 1222-1230.

Yu, S.-m., Wang, J., \& Wang, J.-q. (2017). An interval type-2 fuzzy likelihood-based MABAC approach and its application in selecting hotels on a tourism website. International Journal of Fuzzy Systems, 19(1), 47-61. 
Zadeh. (1965). Fuzzy sets. Information and control, 8(3), 338-353. doi:https://doi.org/10.1016/S00199958(65)90241-X

Zadeh, L. A. (1975). The concept of a linguistic variable and its application to approximate reasoning-I. Information Sciences, 8(3), 199-249.

Zavadskas, E. K., Kaklauskas, A., \& Sarka, V. (1994). The new method of multi-criteria complex proportional assessment of projects. Technological and economic development of economy, 1(3), 131-139.

Zhou, F., \& Chen, T.-Y. (2020). An Integrated Multicriteria Group Decision-Making Approach for Green Supplier Selection Under Pythagorean Fuzzy Scenarios. Ieee Access, 8, 165216165231. 